\title{
Your rugby mates don't need to know your colleagues: Triadic closure with edge colors
}

\author{
Laurent Bulteau $^{\mathrm{a}}$, Niels Grüttemeier ${ }^{\mathrm{b}, *}$, Christian Komusiewicz ${ }^{\mathrm{b}}$, \\ Manuel Sorge ${ }^{c, 1}$ \\ a CNRS, Université Paris-Est Marne-la-Vallée, Paris, France \\ b Fachbereich Mathematik und Informatik, Philipps-Universität Marburg, Marburg, Germany \\ c Faculty of Mathematics, Informatics and Mechanics, University of Warsaw, Warsaw, Poland
}

\section{A R T I C L E I N F O}

\section{Article history:}

Received 25 September 2020

Received in revised form 1 February 2021

Accepted 7 March 2021

Available online 19 March 2021

\section{Keywords:}

Social network analysis

Edge coloring

Exponential time hypothesis

Fixed-parameter tractability

Kernelization

\begin{abstract}
A B S T R A C T
Given an undirected graph $G=(V, E)$ the NP-hard Strong Triadic Closure (STC) problem asks for a labeling of the edges as weak and strong such that at most $k$ edges are weak and for each induced $P_{3}$ in $G$ at least one edge is weak. We study the following generalizations of STC with $c$ different strong edge colors. In Multi-STC an induced $P_{3}$ may receive two strong labels as long as they are different. In Edge-List Multi-STC and Vertex-List Multi-STC we may restrict the set of permitted colors for each edge of $G$. We show that, under the Exponential Time Hypothesis (ETH), Edge-List Multi-STC and Vertex-List Multi-STC cannot be solved in time $2^{o\left(|V|^{2}\right)}$. We proceed with a parameterized complexity analysis in which we extend previous algorithms and kernelizations for STC $[11,14]$ to the three variants or outline the limits of such an extension.
\end{abstract}

(c) 2021 Elsevier Inc. All rights reserved.

\section{Acknowledgment}

We would like to thank the reviewers of Journal of Computer and System Sciences for their helpful comments. In particular, one reviewer pointed out a simpler proof for Theorem $1 \mathrm{~b}$ ).

This study was initiated at the 7th annual research retreat of the Algorithmics and Computational Complexity group of TU Berlin, Darlingerode, Germany, March 18th-23rd, 2018.

\section{Introduction}

Social networks represent relationships between humans such as acquaintance and friendship in online social networks. One task in social network analysis is to determine the strength $[13,34,35,41]$ and type $[6,37,42]$ of the relationship signified

\footnotetext{
A preliminary version of this work appeared in Proceedings of the 11th International Conference on Algorithms and Complexity (CIAC'19) held in Rome, Italy in May 2019. The long version contains full proofs of all statements, and a slightly stronger NP-hardness result for MuLTI-STC with two strong colors.

* Corresponding author.

E-mail addresses: laurent.bulteau@u-pem.fr (L. Bulteau), niegru@informatik.uni-marburg.de (N. Grüttemeier), komusiewicz@informatik.uni-marburg.de (C. Komusiewicz), manuel.sorge@mimuw.edu.pl (M. Sorge).

1 Supported by the People Programme (Marie Curie Actions) of the European Union Seventh Framework Programme (FP7/2007-2013) under REA grant agreement number 631163.11, the Israel Science Foundation (grant no. 551145/14), and by the European Research Council (ERC) under the European Union's Horizon 2020 research and innovation programme under grant agreement number 714704 .

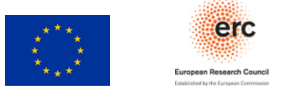


by each edge of the network. One approach to infer strong ties goes back to the notion of strong triadic closure due to Granovetter [12,13] which postulates that, if an agent has strong relations to two other agents, then these two should have at least a weak relation. Following this assertion, Sintos and Tsaparas [35] proposed to find strong ties in social networks by labeling the edges as weak or strong such that the strong triadic closure property is fulfilled and the number of strong edges is maximized.

Sintos and Tsaparas [35] also formulated an extension where agents may have $c$ different types of strong relationships. In this model, the strong triadic closure property only applies to edges of the same strong type. This is motivated by the observation that agents may very well have close relations to agents that do not know each other if these relations themselves arise in segregated contexts. For example, it is quite likely that one's rugby teammates do not know all of one's close colleagues. The edge labellings with up to $c$ strong colors that model this variant of strong triadic closure and the corresponding problem are defined as follows.

Definition 1. A $c$-labeling $L=\left(S_{L}^{1}, \ldots, S_{L}^{c}, W_{L}\right)$ of an undirected graph $G=(V, E)$ is a partition of the edge set $E$ into $c+1$ color classes. The edges in $S_{L}^{i}, i \in\{1, \ldots, c\}$, are strong and the edges in $W_{L}$ are weak; $L$ is an STC-labeling if there exists no pair of edges $\{u, v\} \in S_{L}^{i}$ and $\{v, w\} \in S_{L}^{i}$ such that $\{u, w\} \notin E$. We say that such a pair of edges violates STC for a $c$-labeling understood from the context.

\section{Multi Strong Triadic Closure (Multi-STC)}

Input: An undirected graph $G=(V, E)$ and integers $c \in \mathbb{N}$ and $k \in \mathbb{N}$.

Question: Is there a $c$-colored STC-labeling $L$ with $\left|W_{L}\right| \leq k$ ?

We refer to the special case $c=1$ as Strong Triadic Closure (STC). STC, and thus Multi-STC, is NP-hard [35]. We study the complexity of Multi-STC and two generalizations of MulTi-STC which are defined as follows.

The first generalization deals with the case where one restricts the set of possible relations for some agents. Assume, for example, that strong edges correspond to family relations or professional relations. If one knows the profession of some agents, then this knowledge can be modeled by introducing different strong colors for each profession and constraining the sought edge labeling in such a way that each agent may receive only a strong edge corresponding to a familial relation or to his profession. In other words, for each agent we are given a list of allowed strong colors that may be assigned to incident relationships. Formally, we arrive at the following extension of STC-labellings.

Definition 2. Let $G=(V, E)$ be a graph, $\Lambda: V \rightarrow 2^{\{1,2, \ldots, c\}}$ a mapping for some $c \in \mathbb{N}$, and $L=\left(S_{L}^{1}, \ldots, S_{L}^{c}, W_{L}\right)$ a $c$-colored STC-labeling. We say that an edge $\{v, w\} \in E$ satisfies the $\Lambda$-list property under $L$ if $\{v, w\} \in W_{L}$ or $\{v, w\} \in S_{L}^{\alpha}$ for some $\alpha \in \Lambda(v) \cap \Lambda(w)$. We call a $c$-colored STC-labeling $\Lambda$-satisfying if every edge $e \in E$ satisfies the $\Lambda$-list property under $L$.

\section{Vertex-List Multi Strong Triadic Closure (VL-Multi-STC)}

Input: An undirected graph $G=(V, E)$, integers $c \in \mathbb{N}$ and $k \in \mathbb{N}$, and vertex lists $\Lambda: V \rightarrow 2^{\{1,2, \ldots, c\}}$.

Question: Is there a $\Lambda$-satisfying STC-labeling $L$ with $\left|W_{L}\right| \leq k$ ?

Multi-STC is the special case where $\Lambda(v)=\{1, \ldots, c\}$ for all $v \in V$.

The second generalization deals with the case where one restricts the set of possible relationship types for each relation. For example, if two rugby players live far apart, it is unlikely that they play rugby together. We may model this knowledge by restricting the number of relationship types for this specific relationship. In other words, for each relationship we are given a list of possible strong colors that may be assigned with to this relationship. Observe that this is an actual generalization of VL-MulTi-STC: Consider three agents $v_{1}, v_{2}$, and $v_{3}$ that are pairwise related in the network. Assume the relationship between $v_{1}$ and $v_{2}$ and the relationship between $v_{1}$ and $v_{3}$ are both restricted to 'rugby' and 'colleagues'. If now the relationship between $v_{2}$ and $v_{3}$ is restricted to 'ballet class' and 'drinking buddies', this situation cannot be expressed with vertex lists. This more general constraint is formalized as follows.

Definition 3. Let $G=(V, E)$ be a graph, $\Psi: E \rightarrow 2^{\{1,2, \ldots, c\}}$ a mapping for some value $c \in \mathbb{N}$ and $L=\left(S_{L}^{1}, \ldots, S_{L}^{c}, W_{L}\right)$ a $c$ colored STC-labeling. We say that an edge $e \in E$ satisfies the $\Psi$-list property under $L$ if $e \in W_{L}$ or $e \in S_{L}^{\alpha}$ for some $\alpha \in \Psi(e)$. We call a $c$-colored STC-labeling $\Psi$-satisfying if every edge $e \in E$ satisfies the $\Psi$-list property under $L$.

This leads to the most general problem of this work.

Edge-List Multi Strong Triadic Closure (EL-Multi-STC)

Input: An undirected graph $G=(V, E)$, integers $c \in \mathbb{N}$ and $k \in \mathbb{N}$ and edge lists $\Psi: E \rightarrow 2^{\{1,2, \ldots, c\}}$.

Question: Is there a $\Psi$-satisfying STC-labeling $L$ with $\left|W_{L}\right| \leq k$ ?

From a more abstract point of view, in STC we need to cover all induced $P_{3} \mathrm{~S}$, the paths on three vertices, in a graph by selecting at most $k$ edges, a natural graph-theoretic task. Moreover, as we discuss later, all STC-problems studied here have 
Table 1

An overview of the parameterized complexity results for the parameters number $k$ of weak edges, number $c$ of colors, and number $k_{1}$ of weak edges for $c=1$.

\begin{tabular}{|c|c|c|}
\hline Parameter & Multi-STC & VL-Multi-STC \\
\hline$k$ & \multicolumn{2}{|c|}{ FPT if $c \leq 2$, NP-hard for $k=0$ for all $c \geq 3$} \\
\hline$k_{1}$ & $4 k_{1}$-vertex kernel (Corollary 5) & W[1]-hard (Theorem 4) \\
\hline$\left(c, k_{1}\right)$ & $\begin{array}{r}\mathcal{O}\left((c+1)^{k_{1}} \cdot(\mathrm{cm}\right. \\
4 k_{1} \text {-vertex kernel (Corollary 5) }\end{array}$ & $\begin{array}{l}\text { time (Theorem } 3 \text { ) } \\
\text { no polynomial kernel (Corollary } 4 \text { ) } \\
2^{c+1} k_{1} \text {-vertex kernel (Corollary } 5 \text { ) }\end{array}$ \\
\hline
\end{tabular}

close ties to finding proper vertex colorings in a related graph, the Gallai graph $[10,29,36]$ of the input graph $G$. Finally, in triangle-free graphs every pair of incident edges forms an induced $P_{3}$. Consequently, on triangle-free graphs MulTiSTC is equivalent to the EDGE-Colorable SUBGRAPH problem which asks to determine whether we can delete at most $k$ edges from $G$ such that the resulting graph has a proper edge-coloring with at most $c$ colors. Hence, a study of MuLTISTC and its two proposed generalizations is motivated not only by the known applications of MuLTI-STC in social network analysis [35,20] or plausible applications of the two generalizations, but also from a pure combinatorial and computational complexity point of view.

Related work So far, most algorithmic work has focused on STC [11,14,24,35,25]. For example, STC is NP-hard even on graphs with maximum degree four [24]. Motivated by this NP-hardness, the parameterized complexity of STC was studied. The two main parameters under consideration so far are the number $k$ of weak edges and the number $\ell:=|E|-k$ of strong edges in an STC-labeling with a minimal number of weak edges. The fixed-parameter tractability for $k$ follows from a reduction to Vertex Cover [35]. Moreover, STC admits a $4 k$-vertex kernel [14]. For $\ell$, STC is fixed-parameter tractable but does not admit a polynomial kernel [11,14]. Golovach et al. [11] considered a further generalization of STC where the aim is to color at most $k$ edges weak such that each induced subgraph isomorphic to a fixed graph $F$ has at least one weak edge. Another variant of STC asks for a labeling in which some prespecified communities are connected via strong edges [20,39]. In recent work, it was shown that MulTI-STC admits kernels for parameters that measure the distance of the input graph to a class of low-degree graphs for which MuLTI-STC can be solved in polynomial time [15].

Our results We study the classic, fine-grained, and parameterized complexity of MuLTI-STC and its two generalizations. In a nutshell, we obtain strong hardness results for VL-MuLTI-STC and EL-MULTI-STC, showing that they cannot be solved in $2^{o\left(n^{2}\right)}$ time on $n$-vertex graphs when assuming the Exponential Time Hypothesis (ETH) [21]. On the positive side, we show that previous fixed-parameter tractability and kernelization results for STC $[35,11,14]$ can be extended even to the most general problem EL-MuLTI-STC when $c$ is an additional parameter. In detail, we obtain the following results.

First, we observe that previous results on the EDGE COLORING problem give, for every fixed $c$, a dichotomy of MULTI-STC into NP-hard and polynomial-time solvable instances with respect to the maximum degree of the input graph. In particular, for all $c \geq 3$, MuLTI-STC is NP-hard even if $k=0$. For VL-MulTi-STC and EL-MulTI-STC, we then show that even an algorithm that is single-exponential in the number $n$ of vertices of the input graph is unlikely. More precisely, we show that, assuming the ETH, there is no $2^{o\left(n^{2}\right)}$-time algorithm for VL-MulTi-STC and EL-MulTI-STC even if $k=0$ and $c \in \mathcal{O}(n)$. This result is achieved by a compression of 3-CNF formulas $\phi$ where each variable occurs in a constant number of clauses into graphs with $\mathcal{O}(\sqrt{|\phi|})$ vertices. The NP-hardness of MuLTI-STC even if $k=0$ implies that for all three problems, there is presumably no polynomial-time approximation algorithm. Furthermore, for VL-MulTI-STC and EL-MulTI-STC it is not even possible to compute an approximation within $2^{o\left(n^{2}\right)}$ time unless ETH fails.

We then proceed to a parameterized complexity analysis for the three problems; see Table 1 for an overview. Since all variants are NP-hard even if $k=0$, we consider a structural parameter related to $k$. This parameter, denoted by $k_{1}$, is the minimum number of weak edges needed in an STC-labeling for $c=1$. Thus, if $k_{1}$ is known, then we may immediately accept all instances with $k \geq k_{1}$; in this sense one may assume $k \leq k_{1}$ for MulTI-STC. For VL-MuLTI-STC and EL-MuLTI-STC this is not necessarily true since some lists might be empty which enforces weak edges.

The parameter $k_{1}$ is relevant for two reasons: First, it allows us to determine to which extent the FPT algorithms for STC carry over to MulTi-STC, VL-Multi-STC, and EL-Multi-STC. Second, $k_{1}$ has a structural interpretation: it is the vertex cover number of the Gallai graph of the input graph $G$. We believe that this parameterization is of independent interest and might be useful for other problems. The specific results are as follows. We provide an $\mathcal{O}\left((c+1)^{k_{1}} \cdot(c|E|+|V| \cdot|E|)\right)$ time algorithm for the most general problem, EL-MuLTI-STC. We then use this algorithm to show that MuLTI-STC is fixedparameter tractable when parameterized by $k_{1}$ alone. In contrast, VL-MuLTI-STC and EL-MuLTI-STC parameterized by $k_{1}$ alone are W[1]-hard as we show. Moreover, both problems are unlikely to admit a kernel that is polynomial in $c+k_{1}$. We do, however, obtain a kernel that is exponential in $c$ and polynomial in $k$ by extending the $4 k_{1}$-vertex kernelization [14] from STC. More precisely, we obtain a $2^{c+1} \cdot k_{1}$-vertex kernel for VL-MuLTI-STC and EL-MuLTI-STC. In the case of MULTI-STC the kernelization gives a kernel with at most $4 k_{1}$ vertices, thus extending the linear-vertex kernel from $c=1$ to arbitrary values of $c$. 
This work is organized as follows. In Section 2, we present our notation and specify the relation of STC and its variants to vertex coloring problems in Gallai graphs. This will provide some first running-time upper bounds and explains why $k_{1}$ is a natural structural parameter. In Section 3, we provide the NP-hardness results and the ETH-based lower bound for EL-MulTi-STC and VL-MulTi-STC. In Section 4, we provide fixed-parameter tractability and intractability results, including the kernelization algorithms.

\section{Preliminaries}

Notation We consider undirected graphs $G=(V, E)$ where $n:=|V|$ denotes the number of vertices and $m:=|E|$ denotes the number of edges in $G$. For a vertex $v \in V$ we denote by $N_{G}(v):=\{u \in V \mid\{u, v\} \in E\}$ the open neighborhood of $v$ and by $N_{G}[v]:=N(v) \cup\{v\}$ the closed neighborhood of $v$. For any two vertex sets $V_{1}, V_{2} \subseteq V$, we let $E_{G}\left(V_{1}, V_{2}\right):=\left\{\left\{v_{1}, v_{2}\right\} \in E \mid\right.$ $v_{1} \in V_{1}, v_{2} \in V_{2}$ \} denote the set of edges between $V_{1}$ and $V_{2}$. For any vertex set $V^{\prime} \subseteq V$, we let $E_{G}\left(V^{\prime}\right):=E_{G}\left(V^{\prime}, V^{\prime}\right)$ be the set of edges between the vertices of $V^{\prime}$. We may omit the subscript $G$ if the graph is clear from the context. The subgraph induced by a vertex set $S$ is denoted by $G[S]:=\left(S, E_{G}(S)\right)$. A subset $S \subseteq V$ is called vertex cover in $G$, if every edge $e \in E$ has at least one endpoint in $S$. In the VERTEX COVER problem, the input is an undirected graph $G$ and an integer $k$ and the question is, whether there exists a vertex cover of size at most $k$ in $G$. A proper vertex coloring with $c$ colors for some $c \in \mathbb{N}$ is a mapping $a: V \rightarrow\{1, \ldots, c\}$ such that there is no edge $\{u, v\} \in E$ with $a(u)=a(v)$. Throughout this work we call a $c$-colored STC-labeling $L$ optimal (for a graph $G$ and lists $\Psi$ ) if $L$ is $\Psi$-satisfying and the number of weak edges $\left|W_{L}\right|$ is minimal.

Parameterized complexity In parameterized complexity [5,8,9,31] we measure the running time of algorithms depending on the total input size $n$ and a problem parameter $k$. A problem is called fixed-parameter tractable (FPT) if it can be solved in $f(k) \cdot \operatorname{poly}(n)$ time for some computable function $f$. An important tool in the development of parameterized algorithms is problem kernelization. A problem kernelization is a polynomial-time preprocessing of the input data by data reduction rules. Given an instance $I$ with parameter $k$, the goal is to compute an equivalent instance $I^{\prime}$ with parameter $k^{\prime} \leq k$ in polynomial time where $I^{\prime}$ has size $g(k)$ for some computable function $g$. The instance $I^{\prime}$ is called problem kernel and $g(k)$ is called the size of the kernel. If $g$ is a polynomial, then the problem kernelization is called polynomial.

A parameterized reduction maps any instance $(I, k)$ of some parameterized problem $L$ in FPT time to an equivalent instance $\left(I^{\prime}, k^{\prime}\right)$ of a parameterized problem $L^{\prime}$ such that $k^{\prime} \leq f(k)$ for some computable function $f$. If the reduction can be performed in polynomial time and $f$ is a polynomial, the parameterized reduction is a polynomial parameter transformation. If a problem is $\mathrm{W}[1]$-hard for a parameter $k$, then it is assumed to be not fixed-parameter tractable for $k$. If there is a parameterized reduction from a W[1]-hard problem $L$ parameterized by $k$ to a problem $L^{\prime}$ parameterized by $k^{\prime}$, then the problem $L^{\prime}$ parameterized by $k^{\prime}$ is W[1]-hard. Some problems that are fixed-parameter tractable do not admit a polynomial kernel unless NP $\subseteq$ coNP/poly. By using polynomial parameter transformations we can transfer these kernel lower bounds to other problems [2]. The Exponential Time Hypothesis (ETH) is a standard complexity theoretical conjecture used to prove lower bounds. It implies that 3-CNF-SAT cannot be solved in $2^{o(|\phi|)}$ time where $\phi$ denotes the input formula [21].

Gallai graphs, c-colorable subgraphs, and their relation to STC MULTI-STC can be formulated in terms of so-called Gallai graphs $[10,29,36]$.

Definition 4. Given a graph $G=(V, E)$, the Gallai graph $\tilde{G}=(\tilde{V}, \tilde{E})$ of $G$ is defined by $\tilde{V}:=E$ and $\tilde{E}:=\left\{\left\{e_{1}, e_{2}\right\} \mid\right.$ $e_{1}$ and $e_{2}$ form an induced $P_{3}$ in $\left.G\right\}$.

The Gallai graph of an $n$-vertex and $m$-edge graph has $\mathcal{O}(\mathrm{m})$ vertices and $\mathcal{O}(\mathrm{mn})$ edges. Gallai graphs do have restricted structure in the sense that not every graph is a Gallai graph of some other graph. However, for every graph $H$, there is a Gallai graph which contains $H$ as subgraph [29]. For $c=1$ (in other words for STC), the relation to Gallai graphs is as follows: A graph $G=(V, E)$ has an STC-labeling with at most $k$ weak edges if and only if its Gallai graph has a vertex cover of size at most $k$ [35]. This gives an $\mathcal{O}\left(1.28^{k}+n m\right)$-time algorithm by using the current fastest algorithm for VERTEX Cover [3]. More generally, a graph $G=(V, E)$ has a $c$-colored STC-labeling with at most $k$ weak edges if and only if the Gallai graph of $G$ has a properly $c$-colorable induced subgraph on $m-k$ vertices [35].

In the following, we extend this relation to EL-MuLTI-STC by considering list-colorings of the Gallai graph. The special cases VL-Multi-STC, Multi-STC, and STC nicely embed into the construction. First, let us define the problem that we need to solve in the Gallai graph formally. Given a graph $G=(V, E)$, we call a mapping $\chi: V \rightarrow\{0,1, \ldots, c\}$ a subgraph-ccoloring if there is no edge $\{u, v\} \in E$ with $\chi(u)=\chi(v) \neq 0$. Vertices $v$ with $\chi(v)=0$ correspond to deleted vertices. The LIST-COLORABLE SUbGraph problem is now as follows.

LIST-COLORABLE SUbGRAPH

Input: An undirected graph $G=(V, E)$ and integers $c \in \mathbb{N}, k \in \mathbb{N}$ and lists $\Gamma: V \rightarrow 2^{\{1, \ldots, c\}}$.

Question: Is there a subgraph-c-coloring $\chi: V \rightarrow\{0,1, \ldots, c\}$ with $|\{v \in V \mid \chi(v)=0\}| \leq k$ and $\chi(w) \in \Gamma(w) \cup\{0\}$ for every $w \in V$ ?

EL-Multi-STC and LIST-Colorable Subgraph have the following relationship. 
Proposition 1. An instance $(G, c, k, \Psi)$ of EL-Multi-STC is a yes-instance if and only if $(\tilde{G}, c, k, \Psi)$ is a yes-instance of LISTCOLORABLE SUBGRAPH, where $\tilde{G}$ is the Gallai graph of $G$.

Proof. To prove the proposition we first describe how to transform $c$-colored $\Psi$-satisfying labeling $L$ for $G$ into a coloring $\chi_{L}$ for $\tilde{G}$ that satisfies $\chi_{L}(v) \in \Psi(v) \cup\{0\}$ for every vertex $v$ of $\tilde{G}$ and vice versa, such that the number of weak edges under $L$ and the number of vertices that receive color 0 under $\chi_{L}$ are the same. Afterwards, we show that $L$ is an STC-labeling if and only if $\chi_{L}$ is a subgraph-c-coloring.

For any $c$-colored labeling $L$ we set $\chi_{L}(e):=i$ for each edge in $S_{L}^{i}, 1 \leq i \leq c$, and $\chi_{L}(e)=0$ for each edge in $W_{L}$. By definition, the $c$-colored labeling $\chi$ is $\Psi$-satisfying if and only if $\chi_{L}$ satisfies the list constraints in the LIST-CoLORABLE SUBGRAPH instance, that is, $\chi_{L}(v) \in \Psi(v) \cup\{0\}$ for each vertex $v$. Moreover, the number of weak edges in $L$ is precisely the number of vertices in $\tilde{G}$ that receive color 0 . By symmetric arguments, each subgraph-c-coloring $\chi$ that satisfies $\Psi$ and has $k$ vertices $v$ such that $\chi(v)=0$ defines a $c$-colored labeling $L_{\chi}$ of $G$ that is $\Psi$-satisfying and has $k$ weak edges.

$\Leftrightarrow$ Let $L$ be an STC-labeling. We show that for all adjacent vertices $u$ and $v$ in $\tilde{G}$ either $\chi_{L}(u) \neq \chi_{L}(v)$ or $\chi_{L}(u)=0$ or $\chi_{L}(v)=0$. Assume that $\chi_{L}(u) \neq 0$ and $\chi_{L}(v) \neq 0$. Then, the corresponding edges $u$ and $v$ in $G$ are colored with some strong colors $S_{L}^{i}$ and $S_{L}^{j}$. Since $u$ and $v$ are adjacent in $\tilde{G}, u$ and $v$ form a $P_{3}$ in $G$ and since $L$ is a c-colored STC-labeling, we have $i \neq j$. Thus, $\chi(u) \neq \chi(v)$.

$(\Leftarrow)$ Let $\chi$ be a subgraph-c-coloring. We show that $L_{\chi}$ is an STC-labeling. Consider a pair of incident edges $u$ and $v$ that form a $P_{3}$ in $G$. If $\chi(u)=0$ or $\chi(v)=0$, then one of the two edges is weak in $L_{\chi}$. Otherwise, we have $\chi(u) \neq \chi(v)$ because $u$ and $v$ are adjacent in $\tilde{G}$. Thus, $L_{\chi}$ assigns $u$ and $v$ to different strong colors. Hence, $L_{\chi}$ is an STC-labeling.

The correspondence from Proposition 1 means that we can solve EL-MulTi-STC by solving LisT-Colorable SubGRAPH on the Gallai graph of the input graph. To this end we give a running time bound for LIST-CoLORABLE SUBGRAPH. The algorithm for obtaining this running time is a straightforward dynamic program over subsets. Since we are not aware of any concrete result in the literature implying this running time bound, we provide a proof for the sake of completeness.

Proposition 2. LIST-ColORABLE SUbGRAPh can be solved in $\mathcal{O}\left(3^{n} \cdot c^{2}(n+m)\right)$ time. EL-MulTI-STC can be solved in $\mathcal{O}\left(3^{m} \cdot c^{2} m n\right)$ time.

Proof. We define a dynamic programming table $D$ with entries of the type $D[S, i]$ where $S \subseteq V$ and $i \in\{1, \ldots, c\}$. The aim is to fill $D$ such that for all entries we have $D[S, i]=$ 'true' if there is a subgraph-c-coloring $\chi$ for $G[S]$ such that $\chi(v) \in$ $\{1, \ldots, i\} \cap \Gamma(v)$ for all $v \in S$ and $D[S, i]=$ 'false' otherwise. Then, the instance is a yes-instance if and only if $D[S, c]=$ 'true' for some $S$ such that $|S| \geq n-k$.

The table is initialized for $i=1$ and each $S \subseteq V$ by setting

$$
D[S, 1]:= \begin{cases}\text { 'true' } & \text { if } S \text { is an independent set } \wedge \forall v \in S: 1 \in \Gamma(v), \\ \text { 'false' } & \text { otherwise. }\end{cases}
$$

For $i>1$, the table entries are computed by the recurrence

$$
D[S, i]:=\left\{\begin{array}{rlrl}
\text { 'true' } \quad & \text { if } \exists S^{\prime} \subseteq S \text { such that } & S^{\prime} \text { is an independent set } \\
& \wedge \forall v \in S^{\prime}: i \in \Gamma(v) \\
& \wedge D\left[S \backslash S^{\prime}, i-1\right]=\text { 'true', } \\
\text { 'false' } \quad \text { otherwise. } &
\end{array}\right.
$$

The correctness proof is straightforward and thus omitted. The running time is dominated by the time needed to fill table entries for $i>1$ and can be seen as follows. For each $i \in\{2, \ldots, c\}$ we consider all partitions of $V$ into $S^{\prime}, S \backslash S^{\prime}$, and $V \backslash S$. These are $3^{n}$ many. For each of them, we check in $\mathcal{O}(c \cdot(m+n))$ time whether $S^{\prime}$ is an independent set and whether $i \in \Gamma(v)$ for all $v \in S^{\prime}$.

The running time for EL-Multi-STC follows from Proposition 1 and the fact that the Gallai graph of a graph $G$ with $n$ vertices and $m$ edges has $\mathcal{O}(m)$ vertices and $\mathcal{O}(m n)$ edges.

\section{Hardness results}

\subsection{NP-hardness of Multi-STC}

We first observe that MuLTI-STC is NP-hard for every fixed $c$ even on bounded-degree graphs. It was claimed that MulTISTC is NP-hard for every fixed $c$ since in the Gallai graph this is exactly the NP-hard problem ODD CYCLE TRANSVERSAL (in case of $c=2$ ) or VerTeX $c$-Coloring (in case of $c \geq 3$ ) [35]. It is not known, however, whether these problems are NP-hard on Gallai graphs. Instead, the NP-hardness can be observed from hardness results for EDGE ColoRING. In EDGE ColoRING one is given a graph $G$ and a number of colors $c$ and the question is whether we can assign the colors $1, \ldots, c$ to the edges such that no two incident edges receive the same color. This gives the following dichotomy of the complexity of MULTI-STC on bounded-degree graphs. 


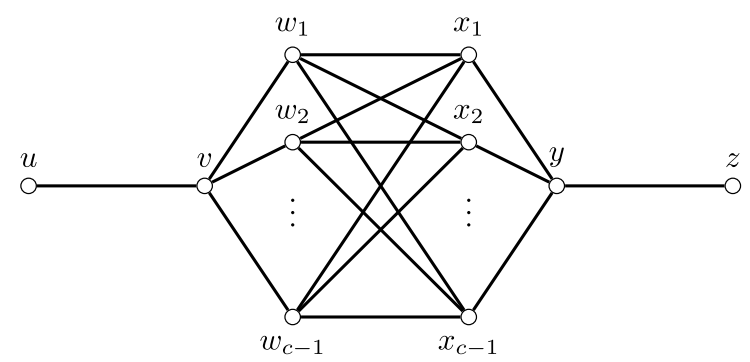

Fig. 1. A graph consisting of edges $\{u, v\},\{y, z\},\left\{v, w_{i}\right\},\left\{y, x_{i}\right\}$, and $\left\{w_{i}, x_{j}\right\}$ for every $i, j \in\{1, \ldots, c-1\}$. It is easy to see that in every proper edge-labeling with colors $1, \ldots, c$, the edges $\{u, v\}$ and $\{y, z\}$ must receive the same color. The graph can thus be used as a gadget to transform an instance of EDGE-LIST Coloring into an equivalent triangle-free instance: we replace every edge $e$ with list $\Psi(e)$ by such gadget, set $\Psi(\{u, v\}):=\Psi(\{y, z\}):=\Psi(e)$, and assign full lists to the other edges of the gadget.

Theorem 1. MuLTI-STC exhibits the following complexity-dichotomy on bounded-degree graphs:

a) For $c=1$, MuLTI-STC is NP-hard on graphs with maximum degree at least four and solvable in polynomial time when the maximum degree is at most three.

b) For $c=2$, MulTI-STC is NP-hard on graphs with maximum degree at least three and solvable in polynomial time when the maximum degree is at most two.

c) For every $c \geq 3$, MuLTI-STC is NP-hard on instances with maximum degree at least c even if $k=0$ and it can be solved in polynomial time if the maximum degree is at most $c-1$.

Proof. Statement $a$ ) is a known result for STC [24]. For statement $c$ ), the NP-hardness follows from the classic result that EDGE Coloring is NP-hard for every fixed $c$ even if the input graph is triangle-free [30]. Furthermore, due to Vizing's Theorem, every graph with maximum degree $\Delta$ can be edge-colored with $\Delta+1$ colors such that no two incident edges receive the same color [40]. Thus, instances with maximum degree at most $c-1$ are trivial yes-instances for MulTI-STC. To show statement $b$ ) we reduce from EDGE Coloring, which is NP-hard even if $c=3$ and the input graph is cubic and triangle-free [18].

Let $I:=(G, c)$ be an instance of EDGE Coloring where $c=3$ and $G$ is a cubic and triangle-free graph. Note that $G$ has an even number of vertices. We define $J:=(G, 2, k)$ with $k:=\frac{n}{2}$ and show that $I$ is a yes-instance of EDGE Coloring if and only if $J$ is a yes-instance of MuLTI-STC.

$(\Rightarrow)$ Let $I$ be a yes-instance of EDGe Coloring. Hence, one can assign the colors 1,2 , and 3 to the edges of $G$ in a way that no two incident edges receive the same color. Then, the fact that $G$ is cubic implies that each color class is a perfect matching in $G$. We let $L:=\left(S_{L}^{1}, S_{L}^{2}, W_{L}\right)$ be a labeling where $S_{L}^{1}$ contains the edges that are assigned with color $1, S_{L}^{2}$ contains the edges that are assigned with color 2 , and $W_{L}$ contains the edges that are assigned with color 3 . Note that $\left|W_{L}\right|=\frac{n}{2}=k$. Furthermore, $L$ is an STC-labeling since no two incident edges in $G$ received the same color. Consequently, $J$ is a yes-instance of MulTi-STC.

$(\Leftarrow)$ Let $J$ be a yes-instance of MuLTI-STC. Then, there exists an STC-labeling $L:=\left(S_{L}^{1}, S_{L}^{2}, W_{L}\right)$ for $G$ such that $\left|W_{L}\right| \leq \frac{n}{2}$. Note that the strong color classes $S_{L}^{1}$ and $S_{L}^{2}$ each form a matching in $G$, since $G$ is triangle-free. We next show that the edges in $W_{L}$ also form a matching. Assume towards a contradiction that there are two edges in $W_{L}$ that share an endpoint. Then, $\left|W_{L}\right| \leq \frac{n}{2}$ implies that there is one vertex $v$ that is not incident with some edge in $W_{L}$. Since $G$ is cubic, this implies that $v$ is incident with two edges of the same strong color. This contradicts the fact that $S_{L}^{1}$ and $S_{L}^{2}$ are matchings in $G$. Consequently, $W_{L}$ is a matching. Then, since the edges of $G$ can be partitioned into three pairwise disjoint matchings, $I$ is a yes-instance of EDGE COLORING.

Note that, for $c=2$, MuLTI-STC parameterized by $k$ is fixed-parameter tractable since we can Solve ODD CYCLE TRANSVERSAL in the Gallai graph $\tilde{G}$ which is fixed-parameter tractable with respect to $k$ [33]. Thus, the dichotomy from Theorem 1 is also tight with regard to the complexity for instances with constant $k$.

\subsection{Fine-grained complexity}

We now provide a stronger hardness result for VL-MulTI-STC and EL-MuLTI-STC: we show that they are unlikely to admit a single-exponential-time algorithm with respect to the number $n$ of vertices. Thus, the simple algorithm behind Proposition 2 is optimal in the sense that $m$ cannot be replaced by $n$ in dense graphs.

We remark that for LIST-EDGE Coloring an ETH-based lower bound of $2^{o\left(|V|^{2}\right)}$ has been shown recently [27]. In LIST-EDGE Coloring one is given a graph $G$, a number $c$ of colors, and a list $\Psi(e)$ of possible colors for each edge $e$. The question is, whether the colors $1,2, \ldots, c$ can be assigned to the edges of $G$ such that no two incident edges receive the same color and each edge receives a color that is on its list. While LIST-EDGE Coloring and EL-MulTI-STC with $k=0$ correspond if the 


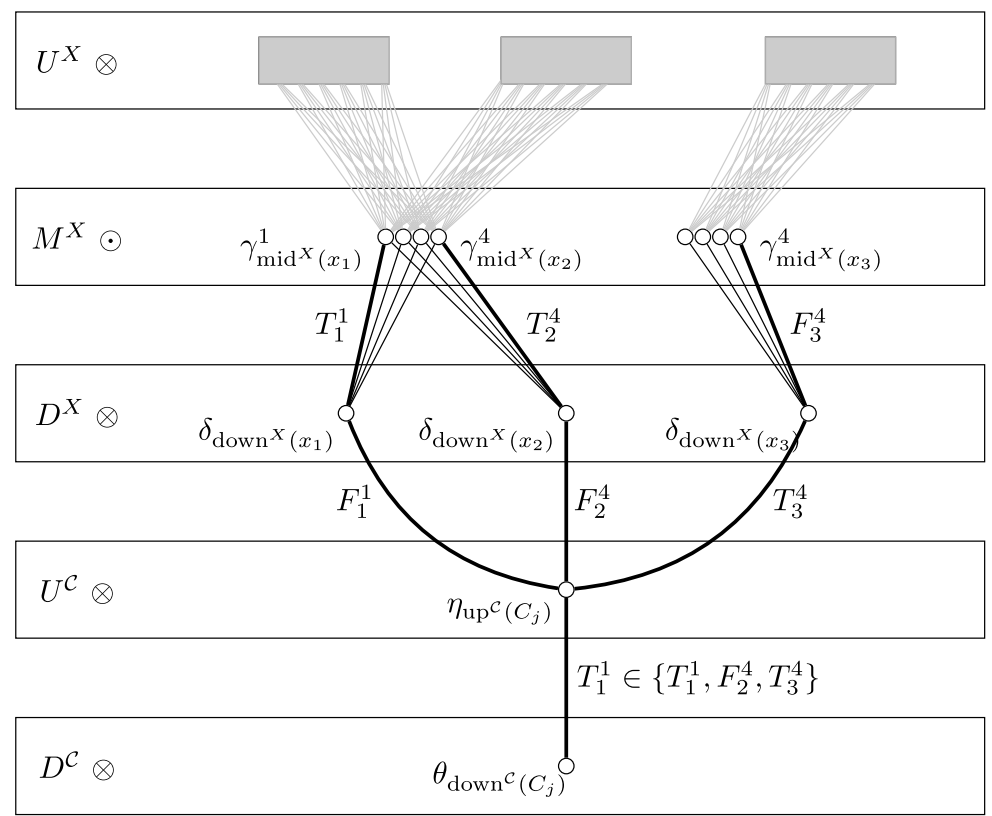

Fig. 2. An example of the construction. The rectangles in $U^{X}$ represent vertices $\alpha_{t}^{\left(r, r^{\prime}\right)}$ with the same value of $t$, $\otimes$ a clique, and $\odot$ an independent set. The edge $\left\{\eta_{\text {up }} \mathcal{C}_{\left(C_{j}\right)}, \theta_{\text {down }} \mathcal{C}_{\left(C_{j}\right)}\right\}$ represents a clause $C_{j}=\left(x_{1} \vee \overline{x_{2}} \vee x_{3}\right)$ with $\Omega\left(C_{j}, x_{1}\right)=1$ and $\Omega\left(C_{j}, x_{2}\right)=\Omega\left(C_{j}, x_{3}\right)=4$. The edge $\left\{\gamma_{\text {mid }}{ }^{X}\left(x_{1}\right)\right.$, $\left.\delta_{\text {down }}{ }_{\left(x_{1}\right)}\right\}$ has strong color $T_{1}^{1}$ which models an assignment where $x_{1}$ is true, which satisfies $C_{j}$. Note that, due to the compression, we may have mid $\left(x_{1}\right)=\operatorname{mid}\left(x_{2}\right)$ and therefore $x_{1}$ and $x_{2}$ may share the four middle vertices.

input graph is triangle-free, the construction behind the lower bound of $2^{o\left(|V|^{2}\right)}$ contains triangles with edge lists that can not be easily modeled with vertex lists. We are not aware of any direct reduction from LIST-EDGE COLORING to VL-MULTI-STC that would transfer the desired lower bound to VL-MULTI-STC.

Note that there is a natural reduction that transforms an instance of LIST-EDGE CoLoRING into an equivalent instance where the input graph is triangle-free: Replace all edges with the gadget shown in Fig. 1. However, this reduction adds $\Omega(\mathrm{cm})$ vertices and therefore does not imply the desired lower bound since the ETH reduction for LIST-EDGE CoLORING outputs a graph where the number of edges is quadratic in the number of vertices [27].

We provide a strong lower bound for VL-MULTI-STC that is based on a reduction from 3-SAT. This reduction is inspired by a reduction used to show that RAINBOW CoLORING cannot be solved in $2^{o\left(n^{3 / 2}\right)}$ time under the ETH [26]. RAINBOw CoLORING is a mildly related problem where the input is a graph and an integer $k$ and the question is, whether the edges can be colored with $k$ distinct colors such that every pair of vertices is connected by a rainbow path, that is, a path where all edges on the path have distinct colors. We remark that for RAINBOw CoLORING another ETH-based lower bound of $2^{o(m)} n^{\mathcal{O}(1)}$ has been shown recently [1]. The compression of the variable part in our reduction works mostly analogously to the reduction to RAInBow Coloring. However, in VL-Multi-STC we have vertex lists that need to be defined carefully. For the clause part of the reduction, we use equitable colorings [17,23] to achieve an even stronger compression and thus a lower bound with a quadratic function in the exponent for VL-MuLTI-STC.

Theorem 2. If the ETH is true, then VL-MuLTI-STC cannot be solved in $2^{o\left(|V|^{2}\right)}$ time even if restricted to instances with $k=0$.

Proof. We give a reduction from 3-SAT to VL-Multi-STC such that the resulting graph has $\mathcal{O}(\sqrt{|\phi|})$ vertices, where $\phi$ is the input formula and $|\phi|$ is the number of variables plus the number of clauses. By the Sparsification Lemma [21], a $2^{o(|\phi|)}$-time algorithm for 3-SAT defeats the ETH and, hence, a $2^{o\left(|V|^{2}\right)}$-time algorithm for VL-MuLTI-STC defeats the ETH as well.

Below, we use $n$ for the number of variables in $\phi$. We can furthermore assume that, in the formula $\phi$, each variable occurs in at most four clauses, since arbitrary 3-CNF formulas can be transformed in polynomial time to an equivalent 3-CNF formula fulfilling this restriction while only increasing the formula length by a constant factor [38]. Observe that in such instances the number of clauses in $\phi$ is at most $\frac{4}{3} n$.

Let $\phi$ be a 3 -CNF formula with a set $X=\left\{x_{1}, \ldots, x_{n}\right\}$ of $n$ variables and a set $\mathcal{C}:=\left\{C_{1}, \ldots, C_{m}\right\}$ of $m \leq \frac{4}{3} n$ clauses. Let $C_{j}$ be a clause and $x_{i}$ a variable occurring in $C_{j}$. We define the occurrence number $\Omega\left(C_{j}, x_{i}\right)$ as the number of clauses in $\left\{C_{1}, C_{2}, \ldots, C_{j}\right\}$ that contain $x_{i}$. Note that $\Omega\left(C_{j}, x_{i}\right)$ is only defined if $x_{i}$ occurs in $C_{j}$. Intuitively, $\Omega\left(C_{j}, x_{i}\right)=r$ means that the $r$ th occurrence of variable $x_{i}$ is the occurrence in clause $C_{j}$. Since each variable occurs in at most four clauses, we have $\Omega\left(C_{j}, x_{i}\right) \in\{1,2,3,4\}$. 


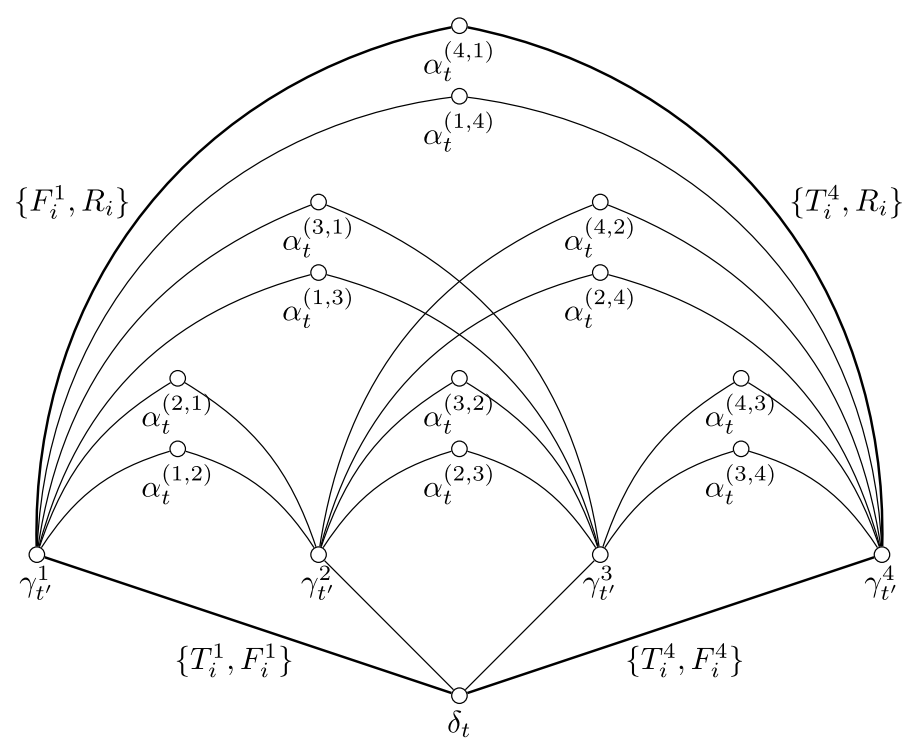

Fig. 3. The variable-representation and the variable-soundness gadget for one variable $x_{i} \in X$ such that $\operatorname{down}^{X}\left(x_{i}\right)=\operatorname{up}^{X}\left(x_{i}\right)=t$ and mid ${ }^{X}\left(x_{i}\right)=t^{\prime}$ with the possible colors for the edges $\left\{\delta_{t}, \gamma_{t^{\prime}}^{1}\right\},\left\{\delta_{t}, \gamma_{t^{\prime}}^{4}\right\},\left\{\alpha_{t}^{(4,1)}, \gamma_{t^{\prime}}^{1}\right\}$, and $\left\{\alpha_{t}^{(4,1)}, \gamma_{t^{\prime}}^{4}\right\}$. Note that labeling $\left\{\delta_{t}, \gamma_{t^{\prime}}^{1}\right\}$ with the strong color $F_{i}^{1}$ and labeling $\left\{\delta_{t}, \gamma_{t^{\prime}}^{4}\right\}$ with the strong color $T_{i}^{4}$ causes a $P_{3}$ with some strong color.

We describe in three steps how to construct an equivalent instance $(G=(V, E), c=9 n+4, k=0, \Lambda)$ for VL-MuLTI-STC such that $|V| \in \mathcal{O}(\sqrt{n})$. First, we describe the variable gadget. Second, we describe the clause gadget. In a third step, we describe how these two gadgets are connected. Before we present the formal construction, we give some intuition.

The strong colors $1, \ldots, 8 n$ represent the truth assignments of the occurrences of the variables. Throughout this proof we refer to these strong colors as $T_{i}^{r}, F_{i}^{r}$ with $i \in\{1, \ldots, n\}$ and $r \in\{1,2,3,4\}$. The idea is that a strong color $T_{i}^{r}$ represents a 'true'-assignment and $F_{i}^{r}$ represents a 'false'-assignment of the $r$ th occurrence of a variable $x_{i} \in X$. The strong colors $8 n+1, \ldots, 9 n+4$ are auxiliary strong colors which we need for the correctness of our construction. Throughout this proof we refer to these strong colors as $R_{1}, \ldots, R_{n}$ and $Z_{1}, Z_{2}, Z_{3}, Z_{4}$. In the variable gadget, there are four distinct edges $e_{1}, e_{2}, e_{3}, e_{4}$ for each variable $x_{i}$ representing the (at most) four occurrences of the variable $x_{i}$. We define vertex lists that ensure that every such edge $e_{r}$ can only be labeled with the strong colors $T_{i}^{r}$ and $F_{i}^{r}$. The coloring of these edges represents a truth assignment to the variable $x_{i}$. In the clause gadget, there are $m$ distinct edges such that the coloring of these edges represents a choice of literals that satisfies $\phi$. The edges between the two gadgets make the values of the literals from the clause part consistent with the assignment of the variable part. The construction consists of five layers of vertices. In the variable gadget we have an upper layer, a middle layer, and a down layer $\left(U^{X}, M^{X}\right.$ and $\left.D^{X}\right)$. In the clause gadget we have an upper and a down layer $\left(U^{\mathcal{C}}\right.$ and $\left.D^{\mathcal{C}}\right)$. Fig. 2 shows a sketch of the construction.

The Variable Gadget. The vertex set of the variable gadget consist of an upper layer, a middle layer and a down layer. The vertices in the middle layer and the down layer form a variable-representation gadget, where each edge between the two parts represents one occurrence of a variable. The vertices in the upper layer form a variable-soundness gadget, which we need to ensure that for each variable either all occurrences are assigned 'true' or all occurrences are assigned 'false'. For an illustration of the variable-representation and the variable-soundness gadget for some variable $x_{i}$ see Fig. 3 .

We start by describing the variable-representation gadget. Let

$$
\begin{array}{rr}
M^{X}: & =\left\{\gamma_{t}^{r} \mid t \in\{1, \ldots,\lceil\sqrt{n}\rceil\}, r \in\{1,2,3,4\}\right\} \quad \text { be the set of middle vertices, and } \\
D^{X}: & =\left\{\delta_{t} \mid t \in\{1, \ldots,\lceil\sqrt{n}\rceil+9\}\right\}
\end{array}
$$

We add edges such that $D^{X}$ becomes a clique in $G$. To specify the correspondence between the variables in $X$ and the edges in the variable-representation gadget, we define below two mappings $\operatorname{mid}^{X}: X \rightarrow\{1, \ldots,\lceil\sqrt{n}\rceil\}$ and down $X: X \rightarrow$ $\{1, \ldots,\lceil\sqrt{n}\rceil+9\}$. Then, for each $x_{i} \in X$ we add four edges $\left\{\gamma_{\operatorname{mid}^{X}\left(x_{i}\right)}^{r}, \delta_{\text {down }^{X}\left(x_{i}\right)}\right\}$ for $r \in\{1,2,3,4\}$. We carefully define the two mappings mid ${ }^{X}$, down ${ }^{X}$ and the vertex lists $\Lambda(v)$ for every $v \in M^{X} \cup D^{X}$ of the variable-representation gadget.

Intuitively, the chosen truth assignment for each variable will be transmitted to a clause by edges between the variable and clause gadgets. To ensure that each such transmitter edge is used for exactly one occurrence of one variable, we first define the variable-conflict graph $H_{\phi}^{X}:=\left(X, \operatorname{Confl}^{X}\right)$ by $\operatorname{Confl}^{X}:=\left\{\left\{x_{i}, x_{j}\right\} \mid x_{i}\right.$ and $x_{j}$ occur in the same clause $\left.C \in \mathcal{C}\right\}$, which we use to define mid $^{X}$ and down ${ }^{X}$. Since every variable of $\phi$ occurs in at most four clauses, the maximum degree of $H_{\phi}^{X}$ is at most 8 . Hence, there is a proper vertex 9-coloring $\chi: X \rightarrow\{1,2, \ldots, 9\}$ for $H_{\phi}^{X}$ which we compute in polynomial time by a folklore greedy algorithm. We end up with 9 color classes $\chi^{-1}(1), \ldots, \chi^{-1}(9)$. Then, we partition each color class $\chi^{-1}(i)$ 
into $\frac{\left|\chi^{-1}(i)\right|}{\lceil\sqrt{n}\rceil}$ groups arbitrarily such that each group has size at most $\lceil\sqrt{n}\rceil$. Let $s$ be the overall number of such groups and let $\mathcal{S}:=\left\{S_{1}, S_{2}, \ldots, S_{S}\right\}$ be the family of all such groups of vertices in $H_{\phi}^{X}$ (each corresponding to a pair of a color $i \in\{1, \ldots, 9\}$ and a group in $\chi^{-1}(i)$ ). The following claim is directly implied by the definition of $\mathcal{S}$ (for part (b) observe that at most $\lceil\sqrt{n}\rceil$ new groups are introduced during the partitioning of the color classes).

Claim 1. For the family $\mathcal{S}:=\left\{S_{1}, S_{2}, \ldots, S_{s}\right\}$ of groups of vertices in $H_{\phi}^{X}$, it holds that

(a) $\left|S_{i}\right| \leq\lceil\sqrt{n}\rceil$ for each $i \in\{1, \ldots, s\}$, and

(b) $s \leq\lceil\sqrt{n}\rceil+9$.

For any given $x_{i} \in X$ we define down ${ }^{X}\left(x_{i}\right):=j$ as the index of the group $S_{j}$ that contains $x_{i}$. The mapping is well-defined since $\mathcal{S}$ forms a partition of the set of variables.

Claim 2. If $x_{i}, x_{j} \in X$ occur in the same clause $C \in \mathcal{C}$, then $\operatorname{down}^{X}\left(x_{i}\right) \neq \operatorname{down}^{X}\left(x_{j}\right)$.

Proof. By definition, $x_{i}$ and $x_{j}$ are adjacent in $H_{\phi}^{X}$. Hence, $x_{i}$ and $x_{j}$ are in different color classes and therefore elements of different groups of $\mathcal{S}$.

Next, we define the mapping $\operatorname{mid}^{X}: X \rightarrow\{1, \ldots,\lceil\sqrt{n}\rceil\}$. To this end, consider the finite sequence Seq ${ }^{n}:=\left(\right.$ down $^{X}\left(x_{1}\right)$, $\left.\operatorname{down}^{X}\left(x_{2}\right), \ldots, \operatorname{down}^{X}\left(x_{n}\right)\right) \in\{1, \ldots,\lceil\sqrt{n}\rceil+9\}^{n}$. We define $\operatorname{mid}^{X}\left(x_{i}\right)$ as the number of occurrences of down ${ }^{X}\left(x_{1}\right)$ in the partial sequence $\operatorname{Seq}^{i}:=\left(\operatorname{down}^{X}\left(x_{1}\right), \ldots, \operatorname{down}^{X}\left(x_{i}\right)\right)$. From Claim 1 (a) we conclude $\operatorname{mid}^{X}\left(x_{i}\right) \in\{1,2, \ldots,\lceil\sqrt{n}\rceil\}$ for every $x_{i} \in X$.

Claim 3. Let $x_{i}, x_{j} \in X$ and $r \in\{1,2,3,4\}$. If $x_{i} \neq x_{j}$, then

$$
\left\{\gamma_{\operatorname{mid}^{X}\left(x_{i}\right)}^{r}, \delta_{\operatorname{down}_{\left(x_{i}\right)}}\right\} \neq\left\{\gamma_{\text {mid }_{\left(x_{j}\right)}^{r}}, \delta_{\operatorname{down}^{X}\left(x_{j}\right)}\right\}
$$

Proof. Without loss of generality, assume $i<j$. Obviously, the claim holds if $\operatorname{down}^{X}\left(x_{i}\right) \neq \operatorname{down}^{X}\left(x_{j}\right)$. Let $\operatorname{down}{ }^{X}\left(x_{i}\right)=$ down ${ }^{X}\left(x_{j}\right)$. Then, there is at least one more occurrence of $\operatorname{down}^{X}\left(x_{i}\right)$ in the partial sequence Seq ${ }_{1}^{j}$ compared to Seq ${ }_{1}^{i}$. Therefore, $\operatorname{mid}^{X}\left(x_{i}\right) \neq \operatorname{mid}^{X}\left(x_{j}\right)$.

Thus we assigned a unique edge in $E\left(M^{X}, D^{X}\right)$ to each occurrence of a variable in $X$. Furthermore, the assigned edges of variables that occur in the same clause do not share an endpoint in $D^{X}$ (Claim 2).

We complete the description of the variable-representation gadget by defining the vertex list $\Lambda(v)$ for every $v \in M^{X} \cup D^{X}$. We set

$$
\begin{array}{lr}
\Lambda\left(\gamma_{t}^{r}\right):=\bigcup_{\substack{x_{i} \in X \\
\operatorname{mid}^{X}\left(x_{i}\right)=t}}\left\{T_{i}^{r}, F_{i}^{r}, R_{i}\right\} & \text { for every } \gamma_{t}^{r} \in M^{X}, \text { and } \\
\Lambda\left(\delta_{t}\right):=\bigcup_{\substack{x_{i} \in X \\
\operatorname{down}^{X}\left(x_{i}\right)=t}}\left\{T_{i}^{1}, T_{i}^{2}, T_{i}^{3}, T_{i}^{4}, F_{i}^{1}, F_{i}^{2}, F_{i}^{3}, F_{i}^{4}, Z_{2}\right\} & \text { for every } \delta_{t} \in D^{X} .
\end{array}
$$

Claim 4. Let $x_{i} \in X$ and $r \in\{1,2,3,4\}$. Then, $\Lambda\left(\gamma_{\operatorname{mid}^{X}\left(x_{i}\right)}^{r}\right) \cap \Lambda\left(\delta_{\operatorname{down}_{\left(x_{i}\right)}^{X}}\right)=\left\{T_{i}^{r}, F_{i}^{r}\right\}$.

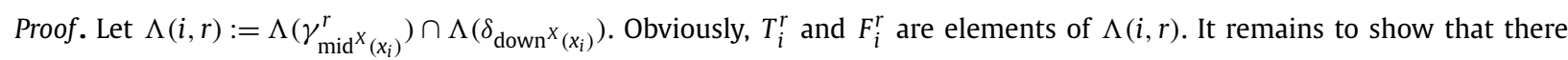
is no other strong color $Y \in \Lambda(i, r)$.

Case 1: $Y=Z_{2}$. Then, $Z_{2} \notin \Lambda\left(\gamma_{t}^{r}\right)$ and it follows that $Y \notin \Lambda(i, r)$.

Case 2: $Y=R_{j}$ with $j \in\{1, \ldots, n\}$. Then, $R_{j} \notin \Lambda\left(\delta_{t}\right)$ and it follows that $Y \notin \Lambda(i, r)$.

Case 3: $Y=T_{j}^{r^{\prime}}$ or $Y=F_{j}^{r^{\prime}}$ with $r^{\prime} \neq r$ and $j \in\{1, \ldots, n\}$. Then, $Y \notin \Lambda\left(\gamma_{\operatorname{mid}^{X}\left(x_{i}\right)}^{r}\right)$ and it follows that $Y \notin \Lambda(i, r)$.

Case 4: $Y=T_{i^{\prime}}^{r}$ or $Y=F_{i^{\prime}}^{r}$ with $i^{\prime} \neq i$. Assuming $T_{i^{\prime}}^{r} \in \Lambda(i, r)$ it follows from the definition of $\Lambda$ that there is some variable $x_{i^{\prime}} \neq x_{i}$ such that $\operatorname{down}^{X}\left(x_{i^{\prime}}\right)=\operatorname{down}^{X}\left(x_{i}\right)$ and $\operatorname{mid}^{X}\left(x_{i^{\prime}}\right)=\operatorname{mid}^{X}\left(x_{i}\right)$, which contradicts Claim 3. Hence, $Y \notin \Lambda(i, r)$.

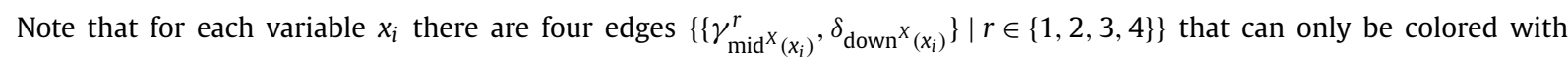
the strong colors $T_{i}^{r}$ and $F_{i}^{r}$ representing the truth assignments of the four occurrences of variable $x_{i}$. We need to ensure that there is no variable $x_{i}$, where, for example, the first occurrence is set to 'true' $\left(T_{i}^{1}\right)$ and the second occurrence is set to 
'false' $\left(F_{i}^{2}\right)$ in a $\Lambda$-satisfying STC-labeling with no weak edges. To this end, we use a variable-soundness gadget, which we describe in the following.

Define

$$
U^{X}:=\left\{\alpha_{t}^{\left(r, r^{\prime}\right)} \mid t \in\{1, \ldots,\lceil\sqrt{n}\rceil+9\},\left(r, r^{\prime}\right) \in\{1,2,3,4\}^{2}, r \neq r^{\prime}\right\}
$$

to be the set of upper vertices. We add edges such that the vertices in $U^{X}$ form a clique in $G$. To specify the correspondence between the variables and the edges in the variable-soundness gadget, we define below a mapping up ${ }^{X}$ : $X \rightarrow\{1,2, \ldots,\lceil\sqrt{n}\rceil+9\}$. The main idea of the variable-soundness gadget is that for each variable $x_{i} \in X$ and each pair $\left\{r, r^{\prime}\right\} \subseteq\{1,2,3,4\}$ there are four edges between the vertices $\gamma_{i}^{r}, \gamma_{i}^{r^{\prime}}$ and the vertices $\alpha_{t}^{\left(r, r^{\prime}\right)}, \alpha_{t}^{\left(r^{\prime}, r\right)}$ of $U^{X}$ which can not all

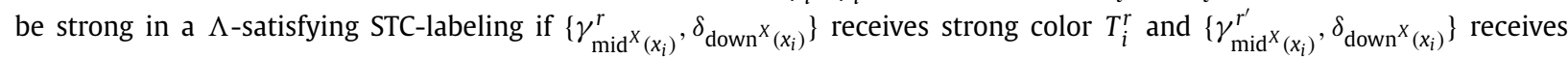
strong color $F_{i}^{r^{\prime}}$. (Recall that we do not allow weak edges.) To this end, we assign a set of 12 endpoints in $U^{X}$ to each variable $x_{i}$. We need to ensure in particular that two variables $x_{i}, x_{j}$ with $\operatorname{mid}^{X}\left(x_{i}\right)=\operatorname{mid}^{X}\left(x_{j}\right)$ do not use the same endpoints in $U^{X}$. We define $\operatorname{up}^{X}\left(x_{i}\right):=\operatorname{down}^{X}\left(x_{i}\right)$. The following is directly implied by Claim 3 .

Claim 5. Let $x_{i}, x_{j} \in X$ with $x_{i} \neq x_{j}$. If $\operatorname{mid}^{X}\left(x_{i}\right)=\operatorname{mid}^{X}\left(x_{j}\right)$, then $\operatorname{up}^{X}\left(x_{i}\right) \neq \operatorname{up}^{X}\left(x_{i}\right)$.

We add the following edges between the vertices of $M^{X}$ and $U^{X}$ : For every variable $x_{i}$, every $r \in\{1,2,3,4\}$, and every $r^{\prime} \in\{1,2,3,4\} \backslash\{r\}$ we add the edges $\left\{\alpha_{\operatorname{up}^{X}\left(x_{i}\right)}^{\left(r, r^{\prime}\right)}, \gamma_{\operatorname{mid}^{X}\left(x_{i}\right)}^{r}\right\}$, and $\left\{\alpha_{\operatorname{up}^{X}\left(x_{i}\right)}^{\left(r, r^{\prime}\right)}, \gamma_{\left.\operatorname{mid}^{X} x_{i}\right)}^{r^{\prime}}\right\}$.

We complete the description of the variable-soundness gadget by defining the vertex lists $\Lambda(v)$ for each $v \in U^{X}$. We set

$$
\Lambda\left(\alpha_{t}^{\left(r, r^{\prime}\right)}\right):=\bigcup_{\substack{x_{i} \in X \\ \operatorname{up}^{X}\left(x_{i}\right)=t}}\left\{T_{i}^{r}, F_{i}^{r^{\prime}}, R_{i}, Z_{1}\right\} \quad \text { for every } \alpha_{t}^{\left(r, r^{\prime}\right)} \in U^{X}
$$

Claim 6. Let $x_{i} \in X$, let $r \in\{1,2,3,4\}$, and let $r^{\prime} \in\{1,2,3,4\} \backslash\{r\}$. Then

a) $\Lambda\left(\alpha_{\operatorname{up}^{X}\left(x_{i}\right)}^{\left(r, r^{\prime}\right)}\right) \cap \Lambda\left(\gamma_{\operatorname{mid}^{X}\left(x_{i}\right)}^{r}\right)=\left\{T_{i}^{r}, R_{i}\right\}$, and

b) $\Lambda\left(\alpha_{\operatorname{up}^{X}\left(x_{i}\right)}^{\left(r, r^{\prime}\right)}\right) \cap \Lambda\left(\gamma_{\operatorname{mid}^{X}\left(x_{i}\right)}^{r^{\prime}}\right)=\left\{F_{i}^{r^{\prime}}, R_{i}\right\}$.

Proof. We first prove statement a). Let $\Lambda\left(i, r, r^{\prime}\right):=\Lambda\left(\alpha_{\operatorname{up}^{X}\left(x_{i}\right)}^{\left(r, r^{\prime}\right)}\right) \cap \Lambda\left(\gamma_{\operatorname{mid}^{X}\left(x_{i}\right)}^{r}\right)$. Clearly, $\Lambda\left(i, r, r^{\prime}\right)$ contains $T_{i}^{r}$ and $R_{i}$. It remains to show that there is no other strong color $Y \in \Lambda\left(i, r, r^{\prime}\right)$. Recall that

$$
\Lambda\left(\gamma_{t}^{r}\right):=\bigcup_{\substack{x_{i} \in X \\ \operatorname{mid}^{X}\left(x_{i}\right)=t}}\left\{T_{i}^{r}, F_{i}^{r}, R_{i}\right\}
$$

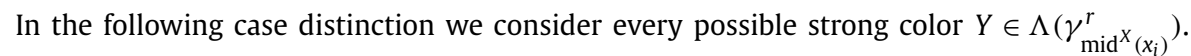

Case a.1: $Y=R_{j}$ or $Y=T_{j}^{r}$ for some $j \neq i$. Then, there is a variable $x_{j} \neq x_{i}$ with mid $^{X}\left(x_{j}\right)=\operatorname{mid}^{X}\left(x_{i}\right)$. It follows by Claim 5 that $\operatorname{up}^{X}\left(x_{j}\right) \neq \operatorname{up}^{X}\left(x_{i}\right)$ and therefore $R_{j}, T_{j}^{r} \notin \Lambda\left(\alpha_{\operatorname{up}^{X}\left(x_{i}\right)}^{\left(r, r^{\prime}\right)}\right)$. Hence, $Y \notin \Lambda\left(i, r, r^{\prime}\right)$.

Case a.2: $Y=F_{j}^{r}$. Then, since

$$
\left\{F_{t}^{p} \mid p \in\{1,2,3,4\}, t \in\{1, \ldots, n\}\right\} \cap \Lambda\left(\alpha_{\operatorname{up}^{X}\left(x_{i}\right)}^{\left(r, r^{\prime}\right)} \subseteq\left\{F_{1}^{r^{\prime}}, F_{2}^{r^{\prime}}, \ldots, F_{n}^{r^{\prime}}\right\} \text { and } r^{\prime} \neq r\right.
$$

we conclude $F_{j}^{r} \notin \Lambda\left(\alpha_{\mathrm{up}^{X}\left(x_{i}\right)}^{\left(r, r^{\prime}\right)}\right)$. Hence, $Y \notin \Lambda\left(i, r, r^{\prime}\right)$.

Next, we prove statement b) with analogous arguments. Let $\bar{\Lambda}\left(i, r, r^{\prime}\right):=\Lambda\left(\alpha_{\operatorname{up}^{X}\left(x_{i}\right)}^{\left(r, r^{\prime}\right)}\right) \Lambda\left(\gamma_{\operatorname{mid}^{X}\left(x_{i}\right)}^{r^{\prime}}\right)$. Clearly, $\left\{F_{i}^{r^{\prime}}, R_{i}\right\} \subseteq$ $\Lambda\left(i, r, r^{\prime}\right)$. It remains to show that there is no other color $Y \in \bar{\Lambda}\left(i, r, r^{\prime}\right)$.

Case b.1: $Y=R_{j}$ or $Y=F_{j}^{r^{\prime}}$ for some $j \neq i$. Then, analogously to Case a.1 we conclude that $Y \notin \bar{\Lambda}\left(i, r, r^{\prime}\right)$.

Case b.2: $Y=T_{j}^{r^{\prime}}$. Then, since

$$
\left\{T_{t}^{p} \mid p \in\{1,2,3,4\}, t \in\{1, \ldots, n\}\right\} \cap \Lambda\left(\alpha _ { \text { up } ^ { X } ( x _ { i } ) } ^ { ( r , r ^ { \prime } ) } \subseteq \left\{\left\{T_{1}^{r}, T_{2}^{r}, \ldots, T_{n}^{r}\right\} \text { and } r^{\prime} \neq r\right.\right.
$$

we conclude $T_{j}^{r^{\prime}} \notin \Lambda\left(\alpha_{\mathrm{up}^{X}\left(x_{i}\right)}^{\left(r, r^{\prime}\right)}\right)$. Hence, $Y \notin \bar{\Lambda}\left(i, r, r^{\prime}\right)$. This completes the proof of Claim 6 . 
This completes the description of the variable gadget. We continue with the description of the clause gadget.

The Clause Gadget. The clause gadget consists of an upper part and a lower part. Let $U^{\mathcal{C}}:=\left\{\eta_{i} \mid i \in\{1, \ldots, 12\lceil\sqrt{n}\rceil+1\}\right\}$ be the set of upper vertices and $D^{\mathcal{C}}:=\left\{\theta_{i} \mid i \in\{1, \ldots,\lceil\sqrt{n}\rceil\}\right\}$ be the set of lower vertices. We add edges such that $U^{\mathcal{C}}$ and $D^{\mathcal{C}}$ each form cliques in $G$.

Recall that for some clause $C_{j} \in \mathcal{C}$ and a variable $x_{i}$ occurring in $C_{j}$ the occurrence number $\Omega\left(C_{j}, x_{i}\right)$ is defined as the number of clauses in $\left\{C_{1}, C_{2}, \ldots, C_{j}\right\}$ that contain $x_{i}$. Below we define two mappings up ${ }^{\mathcal{C}}: \mathcal{C} \rightarrow\{1,2, \ldots, 12\lceil\sqrt{n}\rceil+$ $1\}$, down ${ }^{\mathcal{C}}: \mathcal{C} \rightarrow\{1,2, \ldots,\lceil\sqrt{n}\rceil\}$, and vertex lists $\Lambda: V \rightarrow 2^{\{1, \ldots, c\}}$. Then, for each clause $C_{j} \in \mathcal{C}$ we add an edge $\left\{\eta_{\mathrm{up}^{\mathcal{C}}\left(C_{j}\right)}, \theta_{\text {down }^{\mathcal{C}}\left(C_{j}\right)}\right\}$. Next, we ensure that this edge can only be labeled with the strong colors that match the literals in $C_{j}$. This means, for example, if $C_{j}=\left(x_{1} \vee \overline{x_{2}} \vee x_{3}\right)$ we have $\Lambda\left(\eta_{\mathrm{up}^{\mathcal{C}}\left(C_{j}\right)}\right) \cap \Lambda\left(\theta_{\operatorname{down}^{\mathcal{C}}\left(C_{j}\right)}\right)=\left\{T_{1}^{\Omega\left(C_{j}, x_{1}\right)}, F_{2}^{\Omega\left(C_{j}, x_{2}\right)}, T_{3}^{\Omega\left(C_{j}, x_{3}\right)}\right\}$.

As before, we need to ensure that each variable occurring in a clause has a unique edge between the clause and variable gadgets which transmits the variable's truth assignment to the clause. To achieve this, we define the clause-conflict graph $H_{\phi}^{\mathcal{C}}:=\left(\mathcal{C}, \operatorname{Confl}^{\mathcal{C}}\right)$ by

$$
\begin{aligned}
\operatorname{Confl}^{\mathcal{C}}:=\left\{\left\{C_{i}, C_{j}\right\} \mid\right. & C_{i} \text { contains a variable } x_{i} \text { and } C_{j} \text { contains a variable } x_{j} \\
& \text { such that } \left.\operatorname{down}^{X}\left(x_{i}\right)=\operatorname{down}^{X}\left(x_{j}\right)\right\} .
\end{aligned}
$$

Clauses that share a variable are one example for adjacent vertices in $H_{\phi}^{\mathcal{C}}$. Furthermore, due to the compression there may be distinct variables that are mapped to the same value under down ${ }^{X}$. Two distinct clauses containing these variables are another example for adjacent vertices in $H_{\phi}^{\mathcal{C}}$.

However, from the fact that each variable occurs in at most four clauses in combination with Claim $1 \mathrm{a}$ ), it follows that the maximum degree of $H_{\phi}^{\mathcal{C}}$ is at most $12 \cdot\lceil\sqrt{n}\rceil$. Thus, there exists a proper vertex coloring $\chi: \mathcal{C} \rightarrow\{1,2, \ldots, 12 \cdot\lceil\sqrt{n}\rceil+1\}$ such that each color class $\chi^{-1}(i), i \in\{1, \ldots, 12 \cdot\lceil\sqrt{n}\rceil+1\}$, contains at most $\left\lceil\frac{m}{12 \cdot\lceil\sqrt{n}\rceil+1}\right\rceil+1 \leq\lceil\sqrt{n}\rceil$ clauses [17]. Such coloring is known as equitable coloring and since it has $\mathcal{O}(\sqrt{n})$ colors, it can be computed in polynomial time [23].

For a given clause $C_{i} \in \mathcal{C}$ we define up ${ }^{\mathcal{C}}\left(C_{i}\right):=j$ as the index of the color class $\chi^{-1}(j)$ that contains $C_{i}$. The following claim provides a useful property for the clause gadget and can be shown with similar arguments as Claim 2.

Claim 7. If a clause $C_{j_{1}} \in \mathcal{C}$ contains a variable $x_{i_{1}}$ and a clause $C_{j_{2}} \in \mathcal{C}$ contains a variable $x_{i_{2}}$ such that $\operatorname{down}^{X}\left(x_{i_{1}}\right)=\operatorname{down}^{X}\left(x_{i_{2}}\right)$, then $\operatorname{up}^{\mathcal{C}}\left(C_{j_{1}}\right) \neq \operatorname{up}^{\mathcal{C}}\left(C_{j_{2}}\right)$.

Proof. By definition, $C_{j_{1}}$ and $C_{j_{2}}$ are adjacent in $H_{\phi}^{\mathcal{C}}$. Hence, $C_{j_{1}}$ and $C_{j_{2}}$ are elements of different color classes and therefore $\operatorname{up}^{\mathcal{C}}\left(C_{j_{1}}\right) \neq \operatorname{up}^{\mathcal{C}}\left(C_{j_{2}}\right)$.

Next, we define down ${ }^{\mathcal{C}}$ analogously to up ${ }^{X}$. To this end consider the finite sequence $\operatorname{Seq}^{m}=\left(\operatorname{up}^{\mathcal{C}}\left(C_{1}\right)\right.$, up $^{\mathcal{C}}\left(C_{2}\right), \ldots$, $\left.\operatorname{up}^{\mathcal{C}}\left(C_{m}\right)\right)$ and define down ${ }^{\mathcal{C}}\left(C_{j}\right)$ as the number of occurrences of up $\mathcal{C}^{\mathcal{C}}\left(C_{j}\right)$ in the finite sequence $\operatorname{Seq}^{j}:=\left(\operatorname{up}^{\mathcal{C}}\left(C_{1}\right), \ldots\right.$, $\operatorname{up}^{\mathcal{C}}\left(C_{j}\right)$ ). The fact that each color class contains at most $\lceil\sqrt{n}\rceil$ elements implies down ${ }^{\mathcal{C}}\left(C_{j}\right) \leq\lceil\sqrt{n}\rceil$. Intuitively, the following claim guarantees that distinct clauses correspond to distinct edges, which is an analogue statement to Claim 3.

Claim 8. Let $C_{i}, C_{j} \in \mathcal{C}$. If $C_{i} \neq C_{j}$, then $\left\{\eta_{\operatorname{up}^{\mathcal{C}}\left(C_{i}\right)}, \theta_{\operatorname{down}^{\mathcal{C}}\left(C_{j}\right)}\right\} \neq\left\{\eta_{\mathrm{up}^{\mathcal{C}}\left(C_{j}\right)}, \theta_{\operatorname{down}^{\mathcal{C}}\left(C_{j}\right)}\right\}$.

Proof. Without loss of generality, let $i<j$. The claim obviously holds if $\operatorname{up}^{\mathcal{C}}\left(C_{i}\right) \neq \operatorname{up}^{\mathcal{C}}\left(C_{j}\right)$, so let $\operatorname{up}^{\mathcal{C}}\left(C_{i}\right)=\operatorname{up}^{\mathcal{C}}\left(C_{j}\right)$. Then, there is at least one more occurrence of $\operatorname{up}^{\mathcal{C}}\left(C_{i}\right)$ in the partial sequence $\operatorname{Seq}_{1}^{j}$ compared to $\operatorname{Seq}_{1}^{i}$. Therefore $\operatorname{down}^{\mathcal{C}}\left(C_{i}\right) \neq$ $\operatorname{down}^{\mathcal{C}}\left(C_{j}\right)$.

We complete the description of the clause gadget by defining the vertex lists $\Lambda(v)$ for every $v \in U^{\mathcal{C}} \cup D^{\mathcal{C}}$. For a given clause $C_{j} \in \mathcal{C}$ we define the color set $\mathfrak{X}\left(C_{j}\right)$ and the literal color set $\mathfrak{L}\left(C_{j}\right)$ of $C_{j}$ by

$$
\begin{aligned}
\mathfrak{X}\left(C_{j}\right):= & \left\{T_{i}^{\Omega\left(C_{j}, x_{i}\right)}, F_{i}^{\Omega\left(C_{j}, x_{i}\right)} \mid x_{i} \text { occurs in } C_{j}\right\}, \text { and } \\
\mathfrak{L}\left(C_{j}\right):= & \left\{T_{i}^{\Omega\left(C_{j}, x_{i}\right)} \mid x_{i} \text { occurs as a positive literal in } C_{j}\right\} \cup \\
& \left\{F_{i}^{\Omega\left(C_{j}, x_{i}\right)} \mid x_{i} \text { occurs as a negative literal in } C_{j}\right\} .
\end{aligned}
$$

Note that $\mathfrak{L}\left(C_{j}\right) \subseteq \mathfrak{X}\left(C_{j}\right)$. The vertex lists for the vertices in $U^{\mathcal{C}} \cup D^{\mathcal{C}}$ are defined as

$$
\Lambda\left(\eta_{t}\right):=\bigcup_{\substack{C_{j} \in \mathcal{C} \\ \operatorname{up}^{\mathcal{C}}\left(C_{j}\right)=t}} \mathfrak{X}\left(C_{j}\right) \cup\left\{Z_{3}\right\} \quad \text { for every } \eta_{t} \in U^{\mathcal{C}} \text {, and }
$$




$$
\Lambda\left(\theta_{t}\right):=\bigcup_{\substack{C_{j} \in \mathcal{C} \\ \operatorname{down}^{\mathcal{C}}\left(C_{j}\right)=t}} \mathfrak{L}\left(C_{j}\right) \cup\left\{Z_{4}\right\} \quad \text { for every } \theta_{t} \in D^{\mathcal{C}}
$$

Claim 9. Let $C_{j} \in \mathcal{C}$. Then, $\Lambda\left(\eta_{\mathrm{up}^{\mathcal{C}}\left(C_{j}\right)}\right) \cap \Lambda\left(\theta_{\text {down }^{\mathcal{C}}\left(C_{j}\right)}\right)=\mathfrak{L}\left(C_{j}\right)$.

Proof. Let $\Lambda(j):=\Lambda\left(\eta_{\text {up }^{\mathcal{C}}\left(C_{j}\right)}\right) \cap \Lambda\left(\theta_{\text {down }^{\mathcal{C}}\left(C_{j}\right)}\right)$. Since $\mathfrak{L}\left(C_{j}\right) \subseteq \mathfrak{X}\left(C_{j}\right)$ it holds that $\mathfrak{L}\left(C_{j}\right) \subseteq \Lambda(j)$. It remains to show that there is no other strong color $Y \in \Lambda(j) \backslash \mathfrak{L}\left(C_{j}\right)$.

Case 1: $Y \in\left\{Z_{3}, Z_{4}\right\}$. Then, since $Z_{3} \notin \Lambda\left(\theta_{\text {down }^{\mathcal{C}}\left(C_{j}\right)}\right)$ and $Z_{4} \notin \Lambda\left(\eta_{\mathrm{up}^{\mathcal{C}}\left(C_{j}\right)}\right)$, it follows that $Y \notin \Lambda(j)$.

Case 2: $Y \notin\left\{Z_{3}, Z_{4}\right\}$. Assume towards a contradiction that $Y \in \Lambda(j)$. From $Y \in \Lambda\left(\theta_{\text {down }^{\mathcal{C}}\left(C_{j}\right)}\right)$ it follows that there is a clause $C_{j_{1}}$ with $\operatorname{down}^{\mathcal{C}}\left(C_{j_{1}}\right)=\operatorname{down}^{\mathcal{C}}\left(C_{j}\right)$ and $Y \in \mathfrak{L}\left(C_{j_{1}}\right)$. It holds that $C_{j_{1}} \neq C_{j}$, since otherwise $Y \in \mathfrak{L}\left(C_{j}\right)$, which

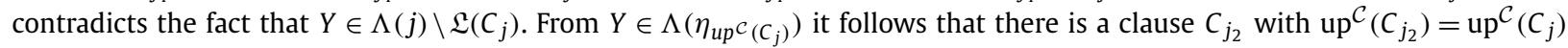
and $Y \in \mathfrak{X}\left(C_{j_{2}}\right)$. By the definition of $\mathfrak{X}$ and $\mathfrak{L}$ there exists a variable $x_{i}$ that occurs in $C_{j_{1}}$ and $C_{j_{2}}$ such that $Y=T_{i}^{\Omega\left(C_{j_{1}}, x_{i}\right)}=$ $T_{i}^{\Omega\left(C_{j_{2}}, x_{i}\right)}$ or $Y=F_{i}^{\Omega\left(C_{j_{1}}, x_{i}\right)}=F_{i}^{\Omega\left(C_{j_{2}}, x_{i}\right)}$. We conclude $\Omega\left(C_{j_{1}}, x_{i}\right)=\Omega\left(C_{j_{2}}, x_{i}\right)$ and therefore $C_{j_{2}}=C_{j_{1}} \neq C_{j}$. Then, the fact that $\operatorname{up}^{\mathcal{C}}\left(C_{j_{1}}\right)=\operatorname{up}^{\mathcal{C}}\left(C_{j}\right)$ and $\operatorname{down}^{\mathcal{C}}\left(C_{j_{2}}\right)=\operatorname{down}^{\mathcal{C}}\left(C_{j}\right)$ contradicts Claim 8 and therefore $Y \notin \Lambda(j)$.

Connecting the Gadgets. We complete the construction of $G$ by describing how the vertices of the variable gadget and the vertices of the clause gadget are connected. The idea is to define edges between the vertices in $D^{X}$ and $U^{\mathcal{C}}$ that model the occurrences of variables in the clauses.

For each $C_{j} \in \mathcal{C}$ we do the following: Let $x_{i_{1}}, x_{i_{2}}$, and $x_{i_{3}}$ be the variables that occur in $C_{j}$. We add the edges: $\left\{\delta_{\text {down }^{X}\left(x_{i_{1}}\right)}, \eta_{\text {up }^{\mathcal{C}}\left(C_{j}\right)}\right\},\left\{\delta_{\text {down }^{X}\left(x_{i_{2}}\right)}, \eta_{\text {up }^{\mathcal{C}}\left(C_{j}\right)}\right\}$, and $\left\{\delta_{\text {down }^{X}\left(x_{i_{3}}\right)}, \eta_{\text {up }^{\mathcal{C}}\left(C_{j}\right)}\right\}$. The idea is that an edge $\left\{\delta_{\text {down }^{X}\left(x_{i}\right)}, \eta_{\text {up }^{\mathcal{C}}\left(C_{j}\right)}\right\} \operatorname{trans}$ mits the truth value of a variable $x_{i}$ to a clause $C_{j}$, where $x_{i}$ occurs as a positive or negative literal. The following claim states that the possible strong colors for such an edge are only $T_{i}^{\Omega\left(C_{j}, x_{i}\right)}$ and $F_{i}^{\Omega\left(C_{j}, x_{i}\right)}$, which correspond to the negated truth assignment of the $\Omega\left(C_{j}, x_{i}\right)$-th occurrence of $x_{i}$.

Claim 10. Let $C_{j} \in \mathcal{C}$ be a clause and let $x_{i} \in X$ be some variable that occurs in $C_{j}$. Then $\Lambda\left(\delta_{\text {down }^{X}\left(x_{i}\right)}\right) \cap \Lambda\left(\eta_{\text {up }}{ }^{\mathcal{C}\left(C_{j}\right)}\right)=$ $\left\{T_{i}^{\Omega\left(C_{j}, x_{i}\right)}, F_{i}^{\Omega\left(C_{j}, x_{i}\right)}\right\}$

Proof. Let $\Lambda(i, j):=\Lambda\left(\delta_{\text {down }^{X}\left(x_{i}\right)}\right) \cap \Lambda\left(\eta_{\mathrm{up}^{\mathcal{C}}\left(C_{j}\right)}\right)$. Obviously, $\left\{T_{i}^{\Omega\left(C_{j}, x_{i}\right)}, F_{i}^{\Omega\left(C_{j}, x_{i}\right)}\right\} \subseteq \Lambda(i, j)$. It remains to show that there is no strong color $Y \in \Lambda(i, j) \backslash\left\{T_{i}^{\Omega\left(C_{j}, x_{i}\right)}, F_{i}^{\Omega\left(C_{j}, x_{i}\right)}\right\}$.

Case 1: $Y=Z_{3}$ or $Y=Z_{2}$. Since $Z_{3} \notin \Lambda\left(\delta_{\text {down }^{X}\left(x_{i}\right)}\right)$ and $Z_{2} \notin \Lambda\left(\eta_{\mathrm{up}^{\mathcal{C}}\left(C_{j}\right)}\right)$, we have $Y \notin \Lambda(i, j)$.

Case 2: $Y=T_{t}^{r}$ or $Y=F_{t}^{r}$ with $t \neq i$ and $r \in\{1,2,3,4\}$. If $Y \notin \Lambda\left(\eta_{\text {up }}{ }_{\left(C_{j}\right)}\right)$, then obviously $Y \notin \Lambda(i, j)$. Thus, let $Y \in$ $\Lambda\left(\eta_{\text {up }^{\mathcal{C}}\left(C_{j}\right)}\right)$. Then, by the definition of $\mathfrak{X}$, there is a clause $C_{j^{\prime}}$ containing a variable $x_{t} \neq x_{i}$ with up $^{\mathcal{C}}\left(C_{j}\right)=$ up $^{\mathcal{C}}\left(C_{j^{\prime}}\right)$. If $C_{j^{\prime}}=$ $C_{j}$, then Claim 2 implies down ${ }^{X}\left(x_{i}\right) \neq \operatorname{down}^{X}\left(x_{t}\right)$ and thus $Y \notin \Lambda\left(\delta_{\operatorname{down}^{X}\left(x_{i}\right)}\right)$. Otherwise, if $C_{j^{\prime}} \neq C_{j}$, then Claim 7 together with the fact that $\operatorname{up}^{\mathcal{C}}\left(C_{j}\right)=\operatorname{up}^{\mathcal{C}}\left(C_{j^{\prime}}\right)$ imply that $\operatorname{down}^{X}\left(x_{i}\right) \neq \operatorname{down}^{X}\left(x_{t}\right)$. Consequently, $Y \notin \Lambda\left(\delta_{\operatorname{down}^{X}\left(x_{i}\right)}\right) \supseteq \Lambda(i, j)$.

Case 3: $Y=T_{i}^{r}$ or $Y=F_{i}^{r}$ with $r \neq \Omega\left(C_{j}, x_{i}\right)$. Obviously, $Y \in \Lambda\left(\delta_{\operatorname{down}^{X}\left(x_{i}\right)}\right)$. Assume towards a contradiction that $Y \in$ $\Lambda\left(\eta_{\text {up }^{\mathcal{C}}\left(C_{j}\right)}\right)$. Then, by the definition of the color set $\mathfrak{X}(\cdot)$, there is a clause $C_{j^{\prime}}$ containing $x_{i}$ such that up ${ }^{\mathcal{C}}\left(C_{j^{\prime}}\right)=$ up $^{\mathcal{C}}\left(C_{j}\right)$ and $\Omega\left(C_{j^{\prime}}, x_{i}\right)=r \neq \Omega\left(C_{j}, x_{i}\right)$. It follows that $C_{j^{\prime}} \neq C_{j}$ which contradicts Claim 7. Hence, $Y \notin \Lambda\left(\eta_{\operatorname{up}^{\mathcal{C}}\left(C_{j}\right)}\right) \supseteq \Lambda(i, j)$.

This completes the description of the construction and basic properties of the VL-MulTi-STC instance $(G, 9 n+4,0, \Lambda)$. Note that $G$ has $\mathcal{O}(\sqrt{n})$ vertices. It remains to show the correctness of the reduction.

Correctness. We show that there is a satisfying assignment for $\phi$ if and only if there is a (9n+4)-colored $\Lambda$-satisfying STC-labeling $L$ for $G$ with strong color classes

$$
S_{L}^{T_{t}^{r}}, S_{L}^{F_{t}^{r}}, S_{L}^{R_{t}}, S_{L}^{Z_{r}} \quad \text { for all } t \in\{1, \ldots, n\} \text { and } r \in\{1,2,3,4\},
$$

and $W_{L}=\emptyset$.

$\Rightarrow$ Let $A: X \rightarrow\{$ 'true', 'false'\} be a satisfying assignment for $\phi$. We describe to which strong color classes we add the edges of $G$ so that we obtain a $\Lambda$-satisfying STC-labeling.

First, we describe to which strong color classes we add the edges of the variable gadget. Formally, these are the edges in $E\left(U^{X} \cup M^{X} \cup D^{X}\right)$. Let $e:=\left\{\delta_{\operatorname{down}^{X}\left(x_{i}\right)}, \gamma_{\text {mid }^{X}\left(x_{i}\right)}^{r}\right\}$ be an edge of the variable-representation gadget for some $x_{i} \in X$ and $r \in$ $\{1,2,3,4\}$. We add $e$ to $S_{L}^{T_{i}^{r}}$ if $A\left(x_{i}\right)=$ 'true' or to $S_{L}^{F_{i}^{r}}$ if $A\left(x_{i}\right)=$ 'false'. In both cases, $e$ satisfies the $\Lambda$-list property by Claim 4. Next, let $e_{1}:=\left\{\gamma_{\operatorname{mid}^{X}\left(x_{i}\right)}^{r}, \alpha_{\operatorname{up}^{X}\left(x_{i}\right)}^{\left(r, r^{\prime}\right)}\right\}$, and $e_{2}:=\left\{\gamma_{\operatorname{mid}^{X}\left(x_{i}\right)}^{r^{\prime}}, \alpha_{\text {up }^{X}\left(x_{i}\right)}^{\left(r, r^{\prime}\right)}\right\}$ be two edges of the variable-soundness gadget for 
some $x_{i} \in X, r \in\{1,2,3,4\}$ and $r^{\prime} \in\{1,2,3,4\} \backslash\{r\}$. We add $e_{1}$ to $S_{L}^{R_{i}}$ if $A\left(x_{i}\right)=$ 'true' or to $S_{L}^{T_{i}^{r}}$ if $A\left(x_{i}\right)=$ 'false'. Further, we add $e_{2}$ to $S_{L}^{F_{i}^{r^{\prime}}}$ if $A\left(x_{i}\right)=$ 'true' or to $S_{L}^{R_{i}}$ if $A\left(x_{i}\right)=$ 'false'. In each case, $e_{1}$ and $e_{2}$ satisfy the $\Lambda$-list property by Claim 6. For the remaining edges of the variable-gadget we do the following: We add all edges of $E\left(U^{X}\right)$ to $S_{L}^{Z_{1}}$ and all edges of $E\left(D_{X}\right)$ to $S_{L}^{Z_{2}}$. Obviously, this does not violate the $\Lambda$-list property.

Second, we describe to which strong color classes we add the edges of the clause gadget. Formally, these are the edges in $E\left(U^{\mathcal{C}} \cup D^{\mathcal{C}}\right)$. Let $C_{j} \in \mathcal{C}$ be a clause. Since $A$ satisfies $\phi$, there is some variable $x_{i}$ occurring in $C_{j}$, such that the assignment $A\left(x_{i}\right)$ satisfies the clause $C_{j}$. Let $r:=\Omega\left(C_{j}, x_{i}\right)$. We add the edge $\left\{\eta_{\mathrm{up}^{\mathcal{C}}\left(C_{j}\right)}, \theta_{\mathrm{down}}{ }^{\mathcal{C}}\left(C_{j}\right)\right\}$ to $S_{L}^{T_{i}^{r}}$ if $A\left(x_{i}\right)=$ 'true' or to $S_{L}^{F_{i}^{r}}$ if $A\left(x_{i}\right)=$ 'false'. In both cases, the edge satisfies the $\Lambda$-list property by Claim 9. For the remaining edges of the clause gadget we do the following: We add all edges of $E\left(U^{\mathcal{C}}\right)$ to $S_{L}^{Z_{3}}$ and all edges of $E\left(D^{\mathcal{C}}\right)$ to $S_{L}^{Z_{4}}$. Obviously, this does not violate the $\Lambda$-list property.

Finally, we describe to which strong color classes we add the edges between the two gadgets. Formally, these are the edges in $E\left(D^{X}, U^{\mathcal{C}}\right)$. Let $C_{j} \in \mathcal{C}$ be a clause and let $x_{i}$ be some variable occurring in $C_{j}$. Let $r:=\Omega\left(C_{j}, x_{i}\right)$. We add the edge

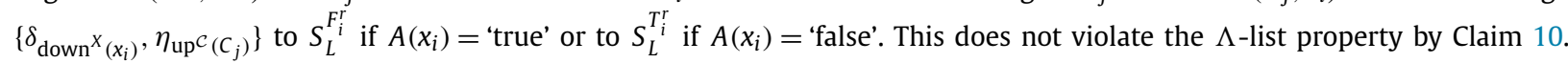

We have now added every edge of $G$ to exactly one strong color class of $L$, such that $L$ is $\Lambda$-satisfying. It remains to show that there is no induced $P_{3}$ containing two edges $\{u, v\}$ and $\{v, w\}$ from the same strong color class. In the following case distinction we consider every possible induced $P_{3}$ on vertices $u, v$, and $w$ where $v$ is the central vertex.

Case 1: $v \in U^{X}$. Then, $v=\alpha_{t}^{\left(r, r^{\prime}\right)}$ for some $t \in\{1, \ldots,\lceil\sqrt{n}\rceil+9\}, r \in\{1,2,3,4\}$ and $r^{\prime} \in\{1,2,3,4\} \backslash\{r\}$. Note that the vertices in $U^{X}$ are not adjacent to vertices in $D^{X}, U^{\mathcal{C}}$ and $D^{\mathcal{C}}$. Thus, it suffices to consider the following subcases.

Case 1.1: $u \in U^{X}$. Then, $\{u, v\} \in S_{L}^{Z_{1}}$. If $w \in U^{X}$, then the vertices $u, v$, and $w$ do not form an induced $P_{3}$, since $U^{X}$ is a clique in $G$. If $w \notin U^{X}$, then $\{v, w\} \notin S_{L}^{Z_{1}}$. Hence, there is no STC-violation.

Case 1.2: $u, w \in M^{X}$. Then, there are variables $x_{i}$ and $x_{j}$ with $\operatorname{up}^{X}\left(x_{i}\right)=\operatorname{up}^{X}\left(x_{j}\right)=t$ and $u=\gamma_{\text {mid }^{X}\left(x_{i}\right)}^{p}, w=\gamma_{\text {mid }^{X}\left(x_{j}\right)}^{q}$ for some $p, q \in\left\{r, r^{\prime}\right\}$. We need to consider the following subcases.

Case 1.2.1: $x_{i} \neq x_{j}$. Then $i \neq j$. By Claim 6 it holds without loss of generality that $\Lambda(u) \cap \Lambda(v) \subseteq\left\{T_{i}^{r}, F_{i}^{r}, T_{i}^{r^{\prime}}, F_{i}^{r^{\prime}}, R_{i}\right\}$ and $\Lambda(w) \cap \Lambda(v) \subseteq\left\{T_{j}^{r}, F_{j}^{r}, T_{j}^{r^{\prime}}, F_{j}^{r^{\prime}}, R_{j}\right\}$. Since $L$ is $\Lambda$-satisfying, the edges $\{u, v\}$ and $\{v, w\}$ are elements of different strong color classes. Thus, there is no STC-violation.

Case 1.2.2: $x_{i}=x_{j}$. Then, $p \neq q$, since otherwise $u=v$. Without loss of generality, we have $u=\gamma_{\text {mid }^{x}\left(x_{i}\right)}^{r}$ and $w=$ $\gamma_{\text {mid }^{x}\left(x_{i}\right)}^{r^{\prime}}$. If $A\left(x_{i}\right)=$ 'true', it follows that $\{u, v\} \in S_{L}^{R_{i}}$ and $\{v, w\} \in S_{L}^{F_{i}^{r^{\prime}}}$. Otherwise, if $A\left(x_{i}\right)=$ 'false', it follows that $\{u, v\} \in$ $S_{L}^{T_{i}^{\prime}}$ and $\{v, w\} \in S_{L}^{R_{i}}$. In both cases, the edges $\{u, v\}$ and $\{v, w\}$ are elements of different strong color classes. Thus, there is no STC-violation.

Case 2: $v \in M^{X}$. Then $v=\gamma_{t}^{r}$ for some $t \in\{1, \ldots,\lceil\sqrt{n}\rceil\}$ and $r \in\{1,2,3,4\}$. Note that the vertices in $M^{X}$ are not adjacent to vertices in $U^{\mathcal{C}}, D^{\mathcal{C}}$ and $M^{X}$. Thus, it suffices to consider the following subcases.

Case 2.1: $u, w \in U^{X}$ or $u, w \in D^{X}$. Then, since $U^{X}$ and $D^{X}$ are cliques in $G$, the vertices $u, v$, and $w$ do not form an induced $P_{3}$ in $G$. Hence, there is no STC-violation.

Case 2.2: $u \in U^{X}$ and $w \in D^{X}$. Then, there are variables $x_{i}$ and $x_{j}$ with $\operatorname{mid}^{X}\left(x_{i}\right)=\operatorname{mid}^{X}\left(x_{j}\right)=t$ and $u \in\left\{\alpha_{\operatorname{up}^{X}\left(x_{i}\right)}^{\left(r, r^{\prime}\right)}, \alpha_{\operatorname{up}^{x}\left(x_{i}\right)}^{\left(r^{\prime}, r\right)}\right\}$, $w=\delta_{\text {down }^{x}\left(x_{j}\right)}$ for some $r^{\prime} \neq r$. We need to consider the following subcases.

Case 2.2.1: $x_{i} \neq x_{j}$. Then, $i \neq j$. Without loss of generality it holds by Claim 6 that $\Lambda(u) \cap \Lambda(v) \subseteq\left\{T_{i}^{r}, F_{i}^{r}, T_{i}^{r^{\prime}}, F_{i}^{r^{\prime}}, R_{i}\right\}$ for some $r^{\prime} \neq r$ and by Claim 4 that $\Lambda(v) \cap \Lambda(w)=\left\{T_{j}^{r}, F_{j}^{r}\right\}$. Since $L$ is $\Lambda$-satisfying, the edges $\{u, v\}$ and $\{v, w\}$ are elements of different strong color classes. Thus, there is no STC-violation.

Case 2.2.2: $x_{i}=x_{j}$. Then, if $A\left(x_{i}\right)=$ 'true' it follows that $\{u, v\} \in S_{L}^{R_{i}} \cup S_{L}^{F_{i}^{r}}$ and $\{v, w\} \in S_{L}^{T_{i}^{r}}$. If $A\left(x_{i}\right)=$ 'false' it follows that $\{u, v\} \in S_{L}^{R_{i}} \cup S_{L}^{T_{i}^{r}}$ and $\{v, w\} \in S_{L}^{F_{i}^{r}}$. In both cases the edges $\{u, v\}$ and $\{v, w\}$ are elements of different strong color classes. Thus, there is no STC-violation.

Case 3: $v \in D^{X}$. Then $v=\delta_{t}$ for some $t \in\{1, \ldots,\lceil\sqrt{n}\rceil+9\}$. Note that the vertices in $D^{X}$ are not adjacent to vertices in $U^{X}$ and $D^{\mathcal{C}}$. Thus, it suffices to consider the following subcases.

Case 3.1: $u \in D^{X}$. Then, $\{u, v\} \in S_{L}^{Z_{2}}$. If $w \in D^{X}$, then the vertices $u, v$, and $w$ do not form an induced $P_{3}$, since $D^{X}$ is a clique in $G$. If $w \notin D^{X}$, then $\{v, w\} \notin S_{L}^{Z_{2}}$. Hence, there is no STC-violation.

Case 3.2: $u, w \in U^{\mathcal{C}}$. Then, the vertices $u, v$, and $w$ do not form an induced $P_{3}$, since $U^{\mathcal{C}}$ forms a clique.

Case 3.3: $u, w \in M^{X}$. By Claim 4, all edges $\{v, y\} \in E\left(\{v\}, M^{X}\right)$ have distinct possible strong colors in $\Lambda(v) \cap \Lambda(y)$. Since $L$ is $\Lambda$-satisfying, the edges $\{u, v\}$ and $\{v, w\}$ are elements of different strong color classes.

Case 3.4: $u \in M^{X}$ and $w \in U^{\mathcal{C}}$. Then, $u=\gamma_{\text {mid }^{X}{ }_{\left(x_{i}\right)}}$ for some $x_{i} \in X$ with down ${ }^{X}\left(x_{i}\right)=t$ and $r \in\{1,2,3,4\}$. Moreover, $w=$ $\eta_{\mathrm{up}}{ }^{\mathcal{B}}\left(C_{j}\right)$ for some clause $C_{j}$ containing a variable $x_{i^{\prime}}$ with $\operatorname{down}^{X}\left(x_{i^{\prime}}\right)=t$. We need to consider the following subcases.

Case 3.4.1: $x_{i} \neq x_{i^{\prime}}$. Then, $i \neq i^{\prime}$ and by Claim 4 we have $\Lambda(u) \cap \Lambda(v)=\left\{T_{i}^{r}, F_{i}^{r}\right\}$ and by Claim 10 we have $\Lambda(v) \cap \Lambda(w)=$ $\left\{T_{i^{\prime}}^{r^{\prime}}, F_{i^{\prime}}^{r^{\prime}}\right\}$ with $r^{\prime}=\Omega\left(C_{j}, x_{i^{\prime}}\right)$. Then, since $L$ is $\Lambda$-satisfying, $\{u, v\}$ and $\{v, w\}$ are not elements of the same strong color class. 
Case 3.4.2: $x_{i}=x_{i^{\prime}}$. Then, if $A\left(x_{i}\right)=$ 'true' it follows that $\{u, v\} \in S_{L}^{T_{i}^{r}}$ and $\{v, w\} \in S_{L}^{F_{i}^{r^{\prime}}}$ for some $r^{\prime} \in\{1,2,3,4\}$. If $A\left(x_{i}\right)=$ 'false', then it follows that $\{u, v\} \in S_{L}^{F_{i}^{r}}$ and $\{v, w\} \in S_{L}^{T_{i}^{r^{\prime}}}$ for some $r^{\prime} \in\{1,2,3,4\}$. In both cases $\{u, v\}$ and $\{v, w\}$ are elements of different strong color classes.

Case 4: $v \in U^{\mathcal{C}}$. Then, $v=\eta_{t}$ for some $t \in\{1, \ldots, 12\lceil\sqrt{n}\rceil+1\}$. Note that the vertices in $U^{\mathcal{C}}$ are not adjacent to vertices in $U^{X}$ and $M^{X}$. Thus, it suffices to consider the following subcases.

Case 4.1: $u \in U^{\mathcal{C}}$. Then, $\{u, v\} \in S_{L}^{Z_{3}}$. If $w \in U^{\mathcal{C}}$, then the vertices $u, v$, and $w$ do not form an induced $P_{3}$ since $U^{X}$ is a clique in $G$. If $w \notin U^{\mathcal{C}}$ it follows that $\{v, w\} \notin S_{L}^{Z_{3}}$. Hence, there is no STC-violation.

Case 4.2: $u, w \in D^{X}$ or $u, w \in D^{\mathcal{C}}$. Then, the vertices $u, v$, and $w$ do not form an induced $P_{3}$, since $D^{X}$ and $D^{\mathcal{C}}$ form cliques in $G$.

Case 4.3: $u \in D^{X}$ and $w \in D^{\mathcal{C}}$. Then, there is a clause $C_{j}$ with up ${ }^{\mathcal{C}}\left(C_{j}\right)=t$ and a clause $C_{j^{\prime}}$ containing a variable $x_{i}$ with $\operatorname{up}^{\mathcal{C}}\left(C_{j^{\prime}}\right)=t$ and $u=\delta_{\operatorname{down}^{X}\left(x_{i}\right)}, w=\theta_{\text {down }^{\mathcal{C}}\left(C_{j}\right)}$. We consider the following subcases.

Case 4.3.1: $C_{j} \neq C_{j^{\prime}}$. Then, since $\operatorname{up}^{\mathcal{C}}\left(C_{j}\right)=\operatorname{up}^{\mathcal{C}}\left(C_{j^{\prime}}\right)$ it follows by Claim 7 that $C_{j}$ and $C_{j^{\prime}}$ do not share a variable. Hence, $x_{i}$ does not occur in $C_{j}$ and therefore $T_{i}^{\Omega\left(C_{j^{\prime}}, x_{i}\right)}, F_{i}^{\Omega\left(C_{j^{\prime}}, x_{i}\right)} \notin \mathfrak{L}\left(C_{j}\right)$. Thus, by Claims 9 and 10 and the fact that $L$ is $\Lambda$-satisfying, the edges $\{u, v\}$ and $\{v, w\}$ are elements of different strong color classes.

Case 4.3.2: $C_{j}=C_{j^{\prime}}$. Let $r:=\Omega\left(C_{j}, x_{i}\right)$. If $\{v, w\} \notin S_{L}^{T_{i}^{r}} \cup S_{L}^{F_{i}^{r}}$, the edges $\{u, v\}$ and $\{v, w\}$ are elements of different color classes. Thus, there is no STC-violation. If $\{v, w\} \in S_{L}^{T_{i}^{r}} \cup S_{L}^{F_{i}^{r}}$ it follows by the construction of $L$ that $C_{j}$ is satisfied by the assignment $A\left(x_{i}\right)$. Without loss of generality assume that $x_{i}$ occurs as a positive literal in $C_{j}$. Then, $A\left(x_{i}\right)=$ 'true'. This implies $\{v, w\} \in S_{L}^{T_{i}^{r}}$ and $\{u, v\} \in S_{L}^{F_{i}^{r}}$. Hence, $\{u, v\}$ and $\{v, w\}$ are elements of different strong color classes.

Case 5: $v \in D^{\mathcal{C}}$. Then, $v$ is not adjacent with any vertices in $U^{X}, M^{X}$ or $D^{X}$. Hence, we need to consider the following cases.

Case 5.1: $u, w \in U^{\mathcal{C}}$ or $u, w \in D^{\mathcal{C}}$. Then, the vertices $u, v$, and $w$ do not form an induced $P_{3}$ since $U^{\mathcal{C}}$ and $D^{\mathcal{C}}$ are cliques in $G$.

Case 5.2: $u \in D^{\mathcal{C}}$ and $w \in U^{\mathcal{C}}$. Then, $\{u, v\} \in S_{L}^{Z_{4}}$ and $\{v, w\} \notin S_{L}^{Z_{4}}$. Hence, there is no STC-violation.

This proves that $L$ is a $\Lambda$-satisfying STC-labeling for $G$ with no weak edges.

$(\Leftarrow)$ Conversely, let $L$ be a $(9 n+4)$-colored $\Lambda$-satisfying STC-labeling for $G$. We show that $\phi$ is satisfiable. We define an assignment $A: C \rightarrow\{$ 'true', 'false' $\}$ by

$$
A\left(x_{i}\right):= \begin{cases}\text { 'true' } & \text { if }\left\{\delta_{\operatorname{down}^{X}\left(x_{i}\right)}, \gamma_{\text {mid }^{X}\left(x_{i}\right)}^{1}\right\} \in S_{L}^{T_{i}^{1}}, \text { and } \\ \text { 'false' } & \text { if }\left\{\delta_{\operatorname{down}^{X}\left(x_{i}\right)}, \gamma_{\text {mid }^{X}\left(x_{i}\right)}^{1}\right\} \in S_{L}^{F_{i}^{1}} .\end{cases}
$$

The assignment is well-defined due to Claim 4. The following claim states that, if one occurrence $r \in\{1,2,3,4\}$ of some variable $x_{i}$ that is assigned 'true' (or 'false', respectively), then so is the first occurrence of $x_{i}$. We obtain this statement by using the variable-soundness gadget.

Claim 11. Let $x_{i} \in X$ and $r \in\{2,3,4\}$.
a) If $\left\{\delta_{\operatorname{down}^{X}\left(x_{i}\right)}, \gamma_{\operatorname{mid}^{X}\left(x_{i}\right)}^{r}\right\} \in S_{L}^{T_{i}^{r}}$, then $\left\{\delta_{\operatorname{down}^{X}\left(x_{i}\right)}, \gamma_{\operatorname{mid}^{X}\left(x_{i}\right)}^{1}\right\} \in S_{L}^{T_{i}^{1}}$.
b) If $\left\{\delta_{\operatorname{down}^{X}\left(x_{i}\right)}, \gamma_{\text {mid }^{X}\left(x_{i}\right)}^{r}\right\} \in S_{L}^{F_{i}^{r}}$, then $\left\{\delta_{\operatorname{down}^{X}\left(x_{i}\right)}, \gamma_{\left.\operatorname{mid}^{X} x_{i}\right)}^{1}\right\} \in S_{L}^{F_{i}^{1}}$.

Proof. We show (a). Let $\left\{\delta_{\operatorname{down}^{X}\left(x_{i}\right)}, \gamma_{\text {mid }^{X}\left(x_{i}\right)}^{r}\right\} \in S_{L}^{T_{i}^{r}}$. Consider the vertex $\alpha_{\operatorname{up}^{X}\left(x_{i}\right)}^{(r, 1)}$. By Claim 6 we have

$$
\begin{aligned}
& \Lambda\left(\alpha_{\operatorname{up}^{X}\left(x_{i}\right)}^{(r, 1)}\right) \cap \Lambda\left(\gamma_{\operatorname{mid}^{X}\left(x_{i}\right)}^{r}\right)=\left\{T_{i}^{r}, R_{i}\right\}, \text { and } \\
& \Lambda\left(\alpha_{\operatorname{up}^{X}\left(x_{i}\right)}^{(r, 1)}\right) \cap \Lambda\left(\gamma_{\operatorname{mid}^{X}\left(x_{i}\right)}^{1}\right)=\left\{F_{i}^{1}, R_{i}\right\} .
\end{aligned}
$$

Note, that the vertices $\delta_{\text {down }^{X}\left(x_{i}\right)}, \gamma_{\operatorname{mid}^{X}\left(x_{i}\right)}^{r}, \alpha_{\text {up }^{X}\left(x_{i}\right)}^{(r, 1)}$ form an induced $P_{3}$ in $G$. Since $L$ is a $\Lambda$-satisfying STC-labeling with no weak edges, it holds that $\left\{\gamma_{\operatorname{mid}^{X}\left(x_{i}\right)}^{r}, \alpha_{\operatorname{up}^{X}\left(x_{i}\right)}^{(r, 1)}\right\} \in S_{L}^{R_{i}}$. Then, since the vertices $\gamma_{\operatorname{mid}_{\left(x_{i}\right)}^{r}}$, $\alpha_{\operatorname{up}^{X}\left(x_{i}\right)}^{(r, 1)}$, and $\gamma_{\text {mid }^{X}\left(x_{i}\right)}^{1}$ form an induced $P_{3}$, the same argument implies $\left\{\alpha_{\mathrm{up}^{X}\left(x_{i}\right)}^{(r, 1)}, \gamma_{\operatorname{mid}^{X}\left(x_{i}\right)}^{1}\right\} \in S_{L}^{F_{i}^{1}}$. Then, since $\Lambda\left(\delta_{\text {down }^{X}\left(x_{i}\right)}\right) \cap \Lambda\left(\gamma_{\text {mid }^{X}\left(x_{i}\right)}^{1}\right)=\left\{T_{i}^{1}, F_{i}^{1}\right\}$ by Claim 4 and the fact that $\delta_{\text {down }^{X}\left(x_{i}\right)}, \gamma_{\operatorname{mid}^{X}\left(x_{i}\right)}^{1}, \alpha_{\text {up }^{x}\left(x_{i}\right)}^{(r, 1)}$ form an induced $P_{3}$ it follows that $\left\{\delta_{\text {down }^{X}\left(x_{i}\right)}, \gamma_{\text {mid }^{X}\left(x_{i}\right)}^{1}\right\} \in S_{L}^{T_{i}^{1}}$ as claimed.

Statement (b) can be shown with the same arguments by considering the vertex $\alpha_{\mathrm{up}^{X}\left(x_{i}\right)}^{(1, r)} \operatorname{instead~of~} \alpha_{\mathrm{up}^{X}\left(x_{i}\right)}^{(r, 1)}$. 
Next we use Claim 11 to show that every clause is satisfied by $A$. Let $C_{j} \in \mathcal{C}$ be a clause. Then, there is an edge $e_{1}:=\left\{\eta_{\text {up }^{\mathcal{C}}\left(C_{j}\right)}, \theta_{\text {down }^{\mathcal{C}}\left(C_{j}\right)}\right\} \in E$. By Claim 9 we have $\Lambda\left(\eta_{\mathrm{up}^{\mathcal{C}}\left(C_{j}\right)}\right) \cap \Lambda\left(\theta_{\text {down }^{\mathcal{C}}\left(C_{j}\right)}\right)=\mathfrak{L}\left(C_{j}\right)$. Since $L$ is $\Lambda$-satisfying, it follows that $e_{1} \in S_{L}^{Y}$ for some $Y \in \mathfrak{L}\left(C_{j}\right)$.

Consider the case $Y=T_{i}^{r}$ for some variable $x_{i}$ that occurs positively in $C_{j}$ and $r=\Omega\left(C_{j}, x_{i}\right)$. We show that $A\left(x_{i}\right)=$ 'true'. Since $x_{i}$ occurs in $C_{j}$ there is an edge $e_{2}:=\left\{\delta_{\text {down }^{x}\left(x_{i}\right)}, \eta_{\text {up }^{\mathcal{C}}\left(C_{j}\right)}\right\} \in E$ which can only be an element of the strong classes $S_{L}^{T_{i}^{r}}$ or $S_{L}^{F_{i}^{r}}$ due to Claim 10. Since $e_{1}$ and $e_{2}$ form an induced $P_{3}$ and $L$ is an STC-labeling we have $e_{2} \in S_{L}^{F_{i}^{r}}$. The edge $e_{3}:=\left\{\delta_{\operatorname{down}^{X}\left(x_{i}\right)}, \gamma_{\operatorname{mid}^{X}\left(x_{i}\right)}^{r}\right\}$ forms an induced $P_{3}$ with $e_{2}$ and can only be an element of the strong classes $S_{L}^{T_{i}^{r}}$ or $S_{L}^{F_{i}^{r}}$ by Claim 4. Hence, $e_{3} \in S_{L}^{T_{i}^{r}}$. By Claim 11 we may conclude $\left\{\delta_{\text {down }^{x}\left(x_{i}\right)}, \gamma_{\operatorname{mid}^{X}\left(x_{i}\right)}^{1}\right\} \in S_{L}^{T_{i}^{1}}$ and therefore $A\left(x_{i}\right)=$ 'true'. Hence, $C_{j}$ is satisfied by $A$.

For the case $Y=F_{i}^{r}$ we can use the same arguments to conclude $A\left(x_{i}\right)=$ 'false'. Hence, $A$ satisfies every clause of $\phi$.

Note that in the instance constructed in the proof of Theorem 2, every edge has at most three possible strong colors and $c \in \mathcal{O}(n)$ where $n$ is the number of variables in $\phi$. This implies the following.

Corollary 1. If the ETH is true, then

a) EL-MuLTI-STC cannot be solved in $2^{o\left(|V|^{2}\right)}$ time even if restricted to instances $(G, c, k, \Psi)$ where $k=0$ and $\max _{e \in E}|\Psi(e)|=3$.

b) VL-MuLTI-STC cannot be solved in $c^{o\left(|V|^{2} / \log c\right)}$ time even if restricted to instances where $k=0$.

Since the lower bound holds for instances with $k=0$, we also obtain a lower bound for approximation algorithms for VL-Multi-STC and EL-Multi-STC.

Corollary 2. If the ETH is true, then there exists no approximation algorithm for VL-MuLTI-STC that runs in time $2^{o\left(n^{2}\right)}$.

\section{Parameterized complexity}

Motivated by the strong hardness results from the previous section, we study the parameterized complexity of MuLTISTC and its list variants. The most natural parameter is the number $k$ of weak edges. The case $c=1$ (STC) is fixed-parameter tractable [35]. For $c=2$, we also obtain an FPT algorithm: An STC-labeling with two strong colors corresponds to a proper two-coloring of the Gallai graph $\tilde{G}$ of the input graph after deleting the vertices corresponding to weak edges. Thus for $c=2$ Multi-STC reduces to OdD CYCLE Transversal in $\tilde{G}$ which is fixed-parameter tractable with respect to $k$ [33]. This extends to EL-MulTI-STC with $c=2$ by modifying the reduction slightly: After computing the Gallai graph, we add two vertex sets $A$ and $B$, each of size $k+1$. Moreover, we add edges such that we obtain a biclique where $A$ and $B$ are the partite sets. We then connect each vertex that may only choose color 1 with every vertex of $A$ and connect each vertex that may only choose color 2 with every vertex of $B$.

In contrast, for every fixed $c \geq 3$, MuLTI-STC is NP-hard even if $k=0$ [30]. Thus, FPT algorithms for the parameters $c, k$, or even the combined parameter $(c, k)$ are unlikely. Instead, we study a structural parameter $k_{1}$ that is related to $k$. Informally, $k_{1}$ is the solution size in the case where $c=1$. Formally, the parameter is defined as follows.

Definition 5. Let $G=(V, E)$ be a graph with a 1-colored STC-labeling $L=\left(S_{L}, W_{L}\right)$ such that there is no 1-colored STClabeling $L^{\prime}=\left(S_{L^{\prime}}, W_{L^{\prime}}\right)$ for $G$ with $\left|W_{L^{\prime}}\right|<\left|W_{L}\right|$. Then we set $k_{1}=k_{1}(G):=\left|W_{L}\right|$.

Equivalently, $k_{1}(G)$ is the size of a minimum vertex cover of the Gallai graph $\tilde{G}$ of $G$ [35].

\subsection{A fixed-parameter algorithm for $\left(c, k_{1}\right)$}

We provide a simple FPT algorithm for EL-MuLTI-STC parameterized by $\left(c, k_{1}\right)$, which is the most general of the three problems. The main idea of the algorithm is to solve LIST-CoLORABLE SUBGRAPH on the Gallai graph of the input graph which is equivalent due to Proposition 1.

Let $(G, c, k, \Psi)$ be an instance of EL-Multi-STC. The first step is to compute the Gallai graph $\tilde{G}=(\tilde{V}, \tilde{E})$ of $G$ which has $m$ vertices and at most $n m$ edges. Observe that $(G, c, k, \Psi)$ is equivalent to the instance $(\tilde{G}, c, k, \Psi)$ of LIST-COLORABLE SUBGrapH due to Proposition 1. We describe an algorithm that solves $(\tilde{G}, c, k, \Psi)$ in $\mathcal{O}\left((c+1)^{s} \cdot(|\tilde{V}| \cdot c+|\tilde{E}|)\right)$ time, where $s=k_{1}$ denotes the size of a minimum vertex cover of $\tilde{G}$.

Let $S \subseteq \tilde{V}$ be a size-s vertex cover of $\tilde{G}$, which can be computed in $\mathcal{O}\left(1.28^{S}+s n\right)$ time $[3]$. Let $I:=\tilde{V} \backslash S$ denote the remaining independent set. We now compute whether $\tilde{G}$ has a subgraph-c-coloring $a: \tilde{V} \rightarrow\{0,1, \ldots, c\}$ with $\mid\{v \in \tilde{V} \mid$ $a(v)=0\} \mid \leq k$.

We enumerate all possible mappings $a_{S}: S \rightarrow\{0,1, \ldots, c\}$. Observe that there are $(c+1)^{S}$ such mappings. For each $a_{S}$ we check whether $a_{S}(v) \in \Psi(v) \cup\{0\}$ for all $v \in S$. Furthermore, we check in $\mathcal{O}(|\tilde{V}| \cdot c+|\tilde{E}|)$ time whether $a_{S}$ is a 

$\{1\}$
$\{1,2\}$
$\{1,2\}$

$\mathrm{O} \longrightarrow$

Fig. 4. A graph $G$ and vertex lists $\Lambda$ of an instance $I:=(G, c=2, k=0, \Lambda)$ of VL-Multi-STC. It is easy to see that $I$ is a no-instance while $k_{1}=1<2=c$.

subgraph-c-coloring for $\tilde{G}[S]$. If this is not the case, then discard the current $a_{S}$. For every other choice of $a_{S}$ we proceed as follows:

We check whether it is possible to extend $a_{S}$ to a mapping $a: \tilde{V} \rightarrow\{0,1, \ldots, c\}$ that is a subgraph-c-coloring for $\tilde{G}$ : For each vertex $v \in I$ we check whether $P_{v}:=\Psi(v) \backslash\left\{a_{S}(w) \mid w \in N_{\tilde{G}}(v)\right\}$ is empty. If $P_{v}=\emptyset$ we set $a(v)=0$. Otherwise, we set $a(v)=p$ for some arbitrary $p \in P_{v}$. This can be done in $\mathcal{O}(|\tilde{V}| \cdot c+|\tilde{E}|)$ time. The resulting mapping $a: V \rightarrow\{0,1, \ldots, c\}$ is a subgraph-c-coloring for $\tilde{G}$, since $a_{S}$ is a subgraph-c-coloring for $G[S]$ and every $v \in I$ has a color $a(v)$ distinct from all vertices in $N(v) \subseteq S$. Finally, we check whether the total number of vertices with $a(v)=0$ is at most $k$. If so, then we accept and stop. Otherwise, we continue with the next mapping $a_{S}$. If we did not accept for any of the enumerated mappings $a_{S}$, then we reject.

The overall running time of the algorithm is $\mathcal{O}\left((c+1)^{s} \cdot(n c+m)\right)$. Recall that $k_{1}=s,|\tilde{V}|=m$ and $|\tilde{E}| \leq n m$. Therefore, we can solve EL-MuLTI-STC in $\mathcal{O}\left((c+1)^{k_{1}} \cdot(c m+n m)\right)$ time.

Lemma 1. The algorithm described above is correct.

Proof. To see that the above algorithm is correct, observe first that it only accepts if it has found a subgraph-c-coloring for $\tilde{G}$ with $|\{v \in \tilde{V} \mid a(v)=0\}| \leq k$. For the other direction, assume that there is a subgraph-c-coloring $a^{\star}: \tilde{V} \rightarrow\{0,1, \ldots, c\}$ with $\left|\left\{v \in \tilde{V} \mid a^{\star}(v)=0\right\}\right| \leq k$. One of the enumerated mappings $a_{S}$ satisfies $a_{S}=\left.a^{\star}\right|_{S}$. For this mapping $a_{S}$ the algorithm sets $a(v)=0$ for some vertex $v \in I$ if and only if $\Psi(v) \backslash\left\{a^{\star}(w) \mid w \in N_{\tilde{G}}(v)\right\}$ is empty. Thus, the number of vertices $v \in \tilde{V}$ with $a(v)=0$ is at most $\left|\left\{v \in \tilde{V} \mid a^{\star}(v)=0\right\}\right| \leq k$, as required.

We obtain the following fixed-parameter tractability result.

Theorem 3. EL-MuLTI-STC can be solved in $\mathcal{O}\left((c+1)^{k_{1}} \cdot(\mathrm{cm}+\mathrm{nm})\right)$ time.

Next, we conclude that MulTI-STC parameterized by $k_{1}$ alone is fixed-parameter tractable. To this end we observe the following relationship between $c$ and $k_{1}$.

Lemma 2. Let $G=(V, E)$ be a graph. For all $c, k \in \mathbb{N}$ with $c>k_{1}(G)$ it holds that $(G, c, k)$ is a yes-instance for MuLTI-STC.

Proof. Let $c>k_{1}$. Then there exists an STC-labeling $L=\left(S_{L}, W_{L}\right)$ for $G$ with one strong color and $\left|W_{L}\right|=k_{1}$. Let $e_{1}, e_{2}, \ldots, e_{k_{1}}$ be the weak edges of $L$. We define a $c$-colored labeling $L^{+}:=\left(S_{L^{+}}^{1}, \ldots, S_{L^{+}}^{c}, W_{L^{+}}\right)$by

$$
W_{L^{+}}:=\emptyset \text { and } S_{L^{+}}^{i}:= \begin{cases}\left\{e_{i}\right\} & \text { for } i \in\left\{1, \ldots, k_{1}\right\}, \\ S_{L} & \text { for } i=k_{1}+1, \\ \varnothing & \text { for } i \in\{k+2, \ldots, c\} .\end{cases}
$$

Since $c>k_{1}$, every edge of $G$ is labeled by $L^{+}$. Because $L$ is an STC-labeling, there is no induced $P_{3}$ containing two edges from $S_{L^{+}}^{k_{1}+1}=S_{L}$. Moreover, since $\left|S_{L^{+}}^{i}\right| \leq 1$ for $i \neq k_{1}+1$, the labeling $L^{+}$satisfies STC. Since $\left|W_{L^{+}}\right|=0$, it holds that $(G, c, k)$ is a yes-instance for MulTi-STC for every $k \in \mathbb{N}$.

Lemma 2 implies an FPT algorithm for MuLTI-STC parameterized by $k_{1}$ alone: Let $(G, c, k)$ be an instance of MuLTI-STC. If $c>k_{1}$, then $(G, c, k)$ is a yes-instance by Lemma 2 . We thus only need to consider instances with $c \leq k_{1}$. Replacing $c$ by $k_{1}$ in the running time bound of Theorem 3 then gives the following.

Corollary 3. MuLTI-STC can be solved in $\mathcal{O}\left(\left(k_{1}+1\right)^{k_{1}} \cdot\left(k_{1} m+n m\right)\right)$ time.

\subsection{W[1]-hardness for vertex-list multi-STC parameterized by $k_{1}$}

The fixed-parameter tractability of MuLTI-STC parameterized by $k_{1}$ alone relies on the relationship between $c$ and $k_{1}$ from Lemma 2. Unfortunately, Lemma 2 does not hold for VL-MuLTI-STC: Fig. 4 shows an example.

We now show that there is little hope to obtain fixed-parameter tractability for the list variants of MuLTI-STC parameterized by $k_{1}$ by giving a parameterized reduction from SET COVER which is defined as follows. 


\section{SET COVER}

Input: A finite universe $U \subseteq \mathbb{N}$, a family $\mathcal{F} \subseteq 2^{U}$, and an integer $t \in \mathbb{N}$.

Question: Is there a subfamily $\mathcal{F}^{\prime} \subseteq \mathcal{F}$ with $\left|\mathcal{F}^{\prime}\right| \leq t$ such that $\bigcup_{F \in \mathcal{F}^{\prime}} F=U$ ?

More precisely, we reduce from SET COVER parameterized by the dual parameter $|\mathcal{F}|-t$. The W[1]-hardness of SET CovER for this parameterization follows from a classic reduction from InDEPENDENT SET [22]. We provide it here for the sake of completeness.

Proposition 3. Set Cover parameterized by $|\mathcal{F}|-t$ is W[1]-hard.

Proof. The Independent Set problem asks for a given graph $G=(V, E)$ and integer $s$ whether there is a subset $V^{\prime} \subseteq V$ of size at least $s$ such that the vertices in $V^{\prime}$ are pairwise non-adjacent in $G$. It is known to be W[1]-hard when parameterized by $s[8]$.

Let $(G=(V, E), s)$ be an instance of Independent Set. We construct a Set Cover-instance $(U, \mathcal{F}, t)$ as follows. Set $U:=E$, $\mathcal{F}:=\left\{F_{v} \mid v \in V\right\}$ with $F_{v}:=\{\{v, u\} \mid u \in N(v)\}$ and $t:=|V|-s$. Note that $|\mathcal{F}|=|V|$, hence $|\mathcal{F}|-t=|V|-(|V|-s)=s$.

Theorem 4. VL-MuLTI-STC parameterized by $k_{1}$ is $W[1]$-hard, even if $k=0$.

Proof. We give a parameterized reduction from SET Cover parameterized by $|\mathcal{F}|-t$ which is W[1]-hard due to Proposition 3. For a given SET Cover-instance $(U, \mathcal{F}, t)$ we describe how to construct an equivalent VL-MulTi-STC-instance $(G=(V, E), c, k, \Lambda)$ with $k_{1} \leq|\mathcal{F}|-t$ and $k=0$. Let $\mathcal{F}=\left\{F_{1}, \ldots, F_{|\mathcal{F}|}\right\}$. Define the vertex set $V$ of the input graph $G$ by $V:=U \cup Z \cup\{a\}$ where $a$ is a new vertex and $Z:=\left\{z_{i} \mid i \in\{t+1, t+2, \ldots,|\mathcal{F}|\}\right\}$. Define the edge set of $G$ by $E:=E_{U} \cup E_{U a} \cup E_{Z a}$ with

- $E_{U}:=\{\{v, w\} \mid v, w \in U\}$,

- $E_{U a}:=\{\{u, a\} \mid u \in U\}$, and

- $E_{Z a}:=\{\{z, a\} \mid z \in Z\}$.

Note that $\left|E_{Z a}\right|=|Z|=|\mathcal{F}|-t$ and that $U$ is a clique.

We let $c:=|\mathcal{F}|+1$ and define the lists $\Lambda$ as

$$
\Lambda(v):= \begin{cases}\left\{i \mid v \in F_{i}\right\} \cup\{|\mathcal{F}|+1\} & \text { if } v \in U, \\ \{1,2, \ldots,|\mathcal{F}|\} & \text { if } v \notin U .\end{cases}
$$

The idea behind this construction is that the vertex $a$ selects sets from $\mathcal{F}$ by labeling the edges in $E_{U a}$. The edges in $E_{Z a}$ ensure, that there are exactly $t$ different strong colors left for the edges in $E_{U a}$.

We first show that $k_{1} \leq|\mathcal{F}|-t$. Let $e_{1}, e_{2} \in E$ be the edges of an induced $P_{3}$ in $G$. Since $U \cup\{a\}$ is a clique by construction, at least one of the edges $e_{1}$ or $e_{2}$ has one endpoint in $Z$, hence it belongs to $E_{Z a}$. Since every $P_{3}$ in $G$ contains at least one edge from $E_{Z a}$ it follows that defining $E_{Z a}$ as weak edges and $E_{U} \cup E_{U a}$ as strong edges yields an STC-labeling with one strong color. This labeling has $\left|E_{Z a}\right|=|\mathcal{F}|-t$ weak edges, hence $k_{1} \leq|\mathcal{F}|-t$.

It remains to show that $(U, \mathcal{F}, t)$ has a solution $\mathcal{F}^{\prime}$ of size $t$ if and only if $G$ has a $\Lambda$-satisfying STC-labeling $L=$ $\left(S_{L}^{1}, \ldots, S_{L}^{|\mathcal{F}|+1}, W_{L}\right)$ with $W_{L}=\emptyset$.

$(\Rightarrow)$ Let $\mathcal{F}^{\prime} \subseteq \mathcal{F}$ be a set cover of $U$ with $\left|\mathcal{F}^{\prime}\right|=t$. Without loss of generality (by renaming) let $\mathcal{F}^{\prime}=\left\{F_{1}, F_{2}, \ldots, F_{t}\right\}$. We define an STC-labeling $L=\left(S_{L}^{1}, \ldots, S_{L}^{|\mathcal{F}|+1}, \emptyset\right)$ as follows. We start by defining the classes $S_{L}^{i}$ for each $i \in\{t+1, t+$ $2, \ldots,|\mathcal{F}|+1\}$. We set

$$
S_{L}^{i}:=\left\{\left\{a, z_{i}\right\}\right\} \text { for every } i \in\{t+1, t+2, \ldots,|\mathcal{F}|\} \text { and } S_{L}^{|\mathcal{F}|+1}:=E_{U} .
$$

Note that $S_{L}^{t+1} \cup \cdots \cup S_{L}^{|\mathcal{F}|+1}=E_{U} \cup E_{Z a}$, so by defining the strong color classes $S_{L}^{t+1}, \ldots, S_{L}^{|\mathcal{F}|+1}$ we have labeled all edges in $E_{U} \cup E_{Z a}$. Before we continue with the definition of $L$, we show that the definition of the strong classes $S_{L}^{t+1}, \ldots, S_{L}^{|\mathcal{F}|+1}$ does not violate the STC property and every edge in $E_{U} \cup E_{Z a}$ satisfies the $\Lambda$-list property. Since $U$ is a clique by construction, there is no induced $P_{3}$ containing two edges from $S_{L}^{|\mathcal{F}|+1}$ violating STC in $E_{U}$. Moreover, since all sets $S_{L}^{t+1}, \ldots, S^{|\mathcal{F}|}$ contain exactly one edge, there is obviously no STC violation in $E_{Z a}$. For every vertex $u \in U$ it holds that $|\mathcal{F}|+1 \in \Lambda(u)$, hence the $\Lambda$-list property is satisfied for every $e \in E_{U}$. Since $\{1,2, \ldots,|\mathcal{F}|\}=\Lambda(a)=\Lambda\left(z_{t+1}\right)=\cdots=\Lambda\left(z_{|\mathcal{F}|}\right)$, the edges in $E_{Z a}$ also satisfy the $\Lambda$-list property.

We now label the edges in $E_{U a}$ by defining the sets $S_{L}^{1}, \ldots, S_{L}^{t}$. Recall that $\mathcal{F}^{\prime}=\left\{F_{1}, \ldots, F_{t}\right\}$ is a set cover of size $t$. We set

$$
S_{L}^{1}:=\left\{\{u, a\} \mid u \in F_{1}\right\} \text { and } S_{L}^{i}:=\left\{\{u, a\} \mid u \in F_{i} \backslash\left(F_{1} \cup \cdots \cup F_{i-1}\right)\right\} \text { for each } i \in\{2,3, \ldots, t\} .
$$


Obviously, each edge of $E_{U a}$ is an element of at most one of the sets $S_{L}^{1}, \ldots, S_{L}^{t}$. Since $\mathcal{F}^{\prime}$ is a set cover, we know that $F_{1} \cup \cdots \cup F_{t}=U$. Hence, $S_{L}^{1}, \ldots, S_{L}^{t}$ is a partition of $E_{U a}$. Since $U \cup\{a\}$ forms a clique, no pair of edges in $E_{U a}$ violates the definition of STC-labellings. Moreover, since the edges in $E_{U a}$ receive different colors from the edges in $E_{Z a}$, no pair of edges from these two sets violates the definition of STC-labellings. From the definition of $\Lambda$ we know that $\Lambda(a)=\{1, \ldots,|\mathcal{F}|\}$ and for every $u \in U$ it holds that $i \in \Lambda(u)$ if $u \in F_{i}$. Hence, every edge in $E_{U a}$ satisfies the $\Lambda$-list property. It follows that $L$ is a $c$-colored STC-labeling with $W_{L}=\emptyset$ such that every edge satisfies the $\Lambda$-list property under $L$.

$(\Leftarrow)$ Conversely, let $L=\left(S_{L}^{1}, \ldots, S_{L}^{|\mathcal{F}|+1}, \emptyset\right)$ be a $c$-colored STC-labeling for $G$ such that every edge of $G$ satisfies the $\Lambda$-list property. We will construct a set cover $\mathcal{F}^{\prime} \subseteq \mathcal{F}$ with $\left|\mathcal{F}^{\prime}\right| \leq t$. We focus on the vertex $a$ and its incident edges. Those are exactly the edges of $E_{U a} \cup E_{Z a}$. Since there are no weak edges, we know that all those edges are elements of strong color classes $S_{L}^{i}$. Since $L$ is an STC-labeling and every pair of edges $e, e^{\prime} \in E_{Z a}$ forms a $P_{3}$, it follows by $\left|E_{Z a}\right|=|\mathcal{F}|-t$ that the edges in $E_{Z a}$ are elements of $|\mathcal{F}|-t$ distinct color classes. Because there is no edge between the vertices of $U$ and $Z$, it also holds that there is no $e \in E_{U a}$ that is an element of the same strong color class as some $e^{\prime} \in E_{Z a}$. Otherwise, $e$ and $e^{\prime}$ form a $P_{3}$ with the same strong color which contradicts the fact that $L$ is an STC-labeling. It follows that the edges in $E_{U a}$ are elements of at most $t$ distinct strong color classes, since $|\Lambda(a)|=|\mathcal{F}|$ and every edge of $G$ satisfies the $\Lambda$-list property under $L$. Without loss of generality (by renaming) we can assume that those strong color classes are $S_{L}^{1}, \ldots, S_{L}^{t}$. Recall that $\mathcal{F}=\left\{F_{1}, F_{2}, \ldots, F_{|F|}\right\}$. We define

$$
\mathcal{F}^{\prime}:=\left\{F_{1}, F_{2}, \ldots, F_{t}\right\}
$$

Obviously, $\left|\mathcal{F}^{\prime}\right|=t$. It remains to show that $\mathcal{F}^{\prime}$ is a set cover. The fact that all edges of $G$ satisfy the $\Lambda$-list property under $L$, implies that for every $u \in U$ there is a $j \in\{1, \ldots, t\}$ such that $j \in \Lambda(u)$. Since $\Lambda(u)=\left\{i \mid u \in F_{i}\right\} \cup\{|\mathcal{F}|+1\}$ for all $u \in U$ by construction, it follows that every $u \in U$ is an element of one of the sets $F_{1}, F_{2}, \ldots, F_{t}$. Hence, $U=F_{1} \cup F_{2} \cup \cdots \cup F_{t}$ and, thus, $\mathcal{F}^{\prime}$ is a set cover of size $t$.

A closer look at the instance $(G, c, k, \Lambda)$ for VL-MulTI-STC constructed from the SET Cover instance $(U, \mathcal{F}, t)$ in the proof of Theorem 4 reveals that $c=|\mathcal{F}|+1$ and $k_{1} \leq|\mathcal{F}|-t$. It follows that $c+k_{1} \leq 2|\mathcal{F}|+1$, so the construction is a polynomial parameter transformation from SET COVER parameterized by $|\mathcal{F}|$ to VL-MuLTI-STC parameterized by $\left(c, k_{1}\right)$. Now, since SET Cover parameterized by $|\mathcal{F}|$ does not admit a polynomial kernel unless NP $\subseteq$ coNP/poly [7] we obtain the following.

Corollary 4. VL-MuLTI-STC parameterized by $\left(c, k_{1}\right)$ does not admit a polynomial kernel unless $N P \subseteq$ coNP/poly.

\subsection{On problem kernelization}

Since EL-MulTI-STC is a generalization of VL-MuLTI-STC, Corollary 4 implies that there is no polynomial kernel for ELMuLTI-STC parameterized by $\left(c, k_{1}\right)$ unless NP $\subseteq$ coNP/poly. We now show that there is a $2^{c+1} \cdot k_{1}$-vertex kernel for ELMulti-STC. To this end, we define a new parameter $\tau$ as follows. Let $I:=(G, c, k, \Psi)$ be an instance of EL-MulTI-STC. Then $\tau:=|\Psi(E) \backslash\{\emptyset\}|$ is defined as the number of different non-empty edge lists occurring in the instance $I$. It clearly holds that $\tau \leq 2^{c}-1$.

For this kernelization we use critical cliques and critical clique graphs [32]. These concepts were also used to obtain linearvertex kernels for graph clustering problems parameterized by the number of edge modifications [16,4] and for STC [14] parameterized by the number of weak edges $k$. Since for STC we can assume that $k=k_{1}$, the kernelization described here lifts this linear-vertex kernel for STC to the more general EL-MuLTI-STC.

Definition 6. A critical clique of a graph $G$ is a clique $K$ where the vertices of $K$ all have the same neighbors in $V \backslash K$, that is, $\forall u, v \in K: N(u) \backslash K=N(v) \backslash K$, and $K$ is maximal under this property. Given a graph $G=(V, E)$, let $\mathcal{K}$ be the collection of its critical cliques. The critical clique graph $\mathcal{C}$ of $G$ is the graph $\left(\mathcal{K}, E_{\mathcal{C}}\right)$ with $\left\{K_{i}, K_{j}\right\} \in E_{\mathcal{C}} \Leftrightarrow \forall u \in K_{i}, v \in K_{j}:\{u, v\} \in E$.

For a critical clique $K$ we let $\mathcal{N}(K):=\bigcup_{K^{\prime} \in N_{\mathcal{C}}(K)} K^{\prime}$ denote the union of its neighbor cliques in the critical clique graph and $\mathcal{N}^{2}(K):=\bigcup_{K^{\prime} \in N_{\mathcal{C}}^{2}(K)} K^{\prime}$ denote the union of the critical cliques at distance exactly two from $K$. The critical clique graph can be constructed in $\mathcal{O}(n+m)$ time [19].

Critical cliques are an important tool for EL-MULTI-STC, because every edge contained in some critical clique is not part of any induced $P_{3}$ in $G$. Hence, each such edge $e$ is strong under any STC-labeling unless $\Psi(e)=\emptyset$. In the following, we will distinguish between two types of critical cliques. We say that a critical clique $K$ is closed if $\mathcal{N}(K)$ forms a clique in $G$ and that $K$ is open otherwise. The number of vertices in open critical cliques is at most $2 k_{1}$ [14]. The main reduction rule of this kernelization describes how to deal with large closed critical cliques. Before we give the concrete rules we provide a useful property of closed critical cliques. Informally, Lemma 3 states that edges between a closed critical clique and a neighbor vertex all behave the same if they have the same strong color lists.

Lemma 3. Let $G=(V, E)$ be a graph, let $K$ be a closed critical clique in $G$, and let $\Psi: E \rightarrow 2^{\{1, \ldots, c\}}$ a mapping for some $c \in \mathbb{N}$. Moreover, let $v \in \mathcal{N}(K)$ and $E^{\prime} \subseteq E(\{v\}, K)$ such that all $e^{\prime} \in E^{\prime}$ have the same strong color list under $\Psi$. Then, there is an optimal STC-labeling $L=\left(S_{L}^{1}, S_{L}^{2}, \ldots, S_{L}^{c}, W_{L}\right)$ for $G$ and $\Psi$ such that $E^{\prime} \subseteq A$ for some $A \in\left\{S_{L}^{1}, \ldots, S_{L}^{c}, W_{L}\right\}$. 


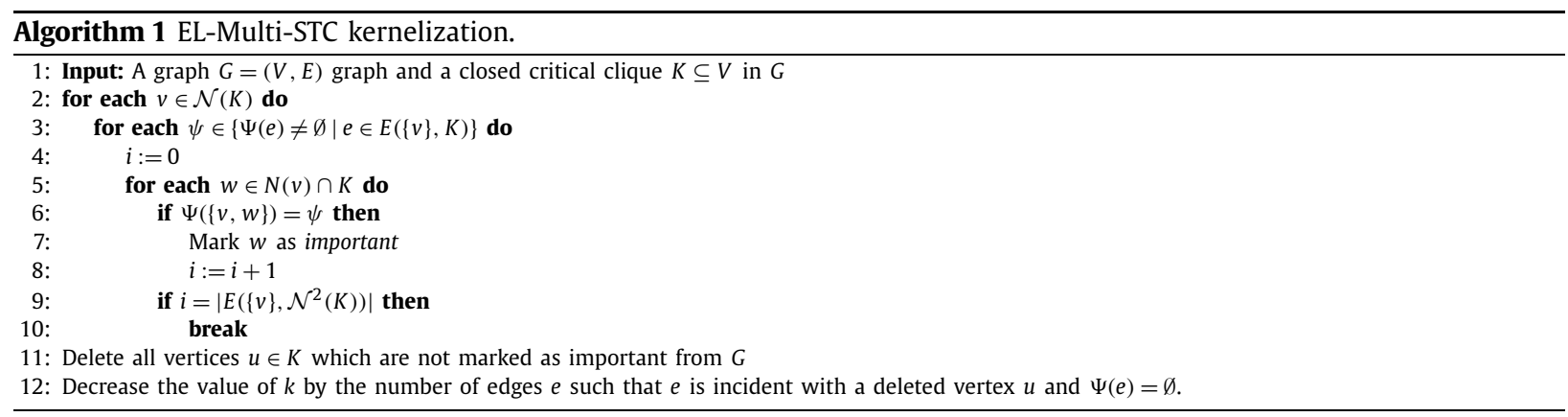

Proof. Pick an optimal STC-labeling $L=\left(S_{L}^{1}, S_{L}^{2}, \ldots, S_{L}^{c}, W_{L}\right)$. If $E^{\prime} \subseteq W_{L}$ nothing more needs to be shown. Otherwise, if $E^{\prime} \nsubseteq W_{L}$, there exists an edge $e \in E^{\prime}$ with $e \in S_{L}^{i}$ for some $i \in\{1, \ldots, c\}$.

We then define a labeling $\hat{L}=\left(S_{\hat{L}}^{1}, S_{\hat{L}}^{2}, \ldots, S_{\hat{L}}^{c}, W_{\hat{L}}\right)$ by $S_{\hat{L}}^{i}:=S_{L}^{i} \cup E^{\prime}, W_{\hat{L}}:=W_{L} \backslash E^{\prime}$ and $S_{\hat{L}}^{j}:=S_{L}^{j} \backslash E^{\prime}$ for $j \neq i$ and show that $\hat{L}$ is a $\Psi$-satisfying optimal STC-labeling.

Let $\bar{e} \in E$ be an edge that forms an induced $P_{3}$ with some edge in $E^{\prime}$. Since $K \cup \mathcal{N}(K)$ forms a clique by the definition of closed critical cliques, it follows that $\bar{e} \in E\left(\{v\}, \mathcal{N}^{2}(K)\right)$. Note that $\bar{e}$ also forms an induced $P_{3}$ with $e$. Then, $\bar{e} \notin S_{\hat{L}}^{i}$ since $L$ is an STC-labeling and $e \in S_{L}^{i}$. Hence, $\hat{L}$ does not violate STC. Moreover, it holds that $\left|W_{\hat{L}}\right| \leq\left|W_{L}\right|$ and thus, $\hat{L}$ is optimal.

From the definition of $E^{\prime}$ and the fact that $e \in S_{L}^{i}$, we know that $i \in \Psi\left(e^{\prime}\right)$ for all $e^{\prime} \in E^{\prime}$. Hence, $\hat{L}$ is $\Psi$-satisfying.

We now may use Lemma 3 in the following way. We take a critical clique $K$ and for each vertex $v \in \mathcal{N}(K)$ and each color list we take an edge between $v$ and $K$ with that color list and we mark the other end of that edge. Then we remove all vertices in $K$ that are not marked, see Algorithm 1. The remaining vertices (and edges) represent the deleted vertices and using Lemma 3 we can show that the deleted edges behave the same as the remaining ones. This gives rise to the following reduction rule.

Rule 1. If $G$ has a closed critical clique $K$ with $|K|>\tau \cdot\left|E\left(\mathcal{N}(K), \mathcal{N}^{2}(K)\right)\right|$, then apply Algorithm 1 on $G$ and $K$.

Proposition 4. Rule 1 is safe and can be applied in polynomial time.

Proof. Let $(G=(V, E), c, k, \Psi)$ be an instance for EL-Multi-STC and let $K$ be a closed critical clique. We show that Algorithm 1 applied on $G$ and $K$ runs in $\mathcal{O}\left(n^{3}\right)$ time and produces an equivalent instance $\left(G^{\prime}=\left(V^{\prime}, E^{\prime}\right), c, k^{\prime}, \Psi^{\prime}\right)$ for ELMulti-STC.

Since $|\mathcal{N}(K)|,|N(v) \cap K| \leq n$ and $|\{\Psi(e) \neq \emptyset \mid e \in E(\{v\}, K)\}| \leq|K| \leq n$, the given algorithm clearly runs in $\mathcal{O}\left(n^{3}\right)$ time. It remains to show that the produced instance $I^{\prime}:=\left(G^{\prime}=\left(V^{\prime}, E^{\prime}\right), c, k^{\prime}, \Psi^{\prime}\right)$ is equivalent to $I:=(G=(V, E), c, k, \Psi)$. Let $D_{V} \subseteq V$ be the set of vertices that were deleted by Algorithm 1 , let $D_{E}$ be the set of edges that are incident with some $v \in \bar{D}_{V}$ and let $D_{E}^{\emptyset} \subseteq D_{E}$ be the set of edges $e \in D_{E}$ with $\Psi(e)=\emptyset$. We have

$$
G^{\prime}=\left(V \backslash D_{V}, E \backslash D_{E}\right) ; k^{\prime}=k-\left|D_{E}^{\emptyset}\right| ; \text { and } \Psi^{\prime}=\left.\Psi\right|_{E \backslash D_{E}} .
$$

We also define $K^{\prime}:=K \backslash D_{V}$ as the modified critical clique in $G^{\prime}$.

$\Leftrightarrow)$ Let $L=\left(S_{L}^{1}, S_{L}^{2}, \ldots, S_{L}^{c}, W_{L}\right)$ be a $\Psi$-satisfying STC-labeling for $G$ such that $\left|W_{L}\right| \leq k$. We define a labeling $\hat{L}=$ $\left(S_{\hat{L}}^{1}, \ldots, S_{\hat{L}}^{c}, W_{\hat{L}}\right)$ by $W_{\hat{L}}:=W_{L} \backslash D_{E}$ and $S_{\hat{L}}^{i}:=S_{L}^{i} \backslash D_{E}$ for each $i \in\{1, \ldots, c\}$. The fact that $L$ is $\Psi$-satisfying implies that $\hat{L}$ is $\Psi^{\prime}$-satisfying. It also holds that

$$
\left|W_{\hat{L}}\right|=\left|W_{L} \backslash D_{E}\right|=\left|W_{L}\right|-\left|W_{L} \cap D_{E}\right| \leq k-\left|D_{E}^{\emptyset}\right|=k^{\prime},
$$

since $D_{E}^{\emptyset} \subseteq W_{L} \cap D_{E}$. It remains to prove that $\hat{L}$ does not violate STC. Assume there is an induced $P_{3}$ on vertices $u, v$, and $w$ in $V^{\prime}$ with edges $\{u, v\},\{v, w\} \in S_{\hat{L}}^{i}$ for some $1 \leq i \leq c$. It follows that $\{u, w\} \in D_{E}$, since $L$ is an STC-labeling. Then, by the definition of $D_{E}$, at least one of the vertices $u$ or $w$ was deleted by the algorithm. This contradicts the fact that $u, w \in V^{\prime}=V \backslash D_{V}$. It follows that $\hat{L}$ is a $\Psi^{\prime}$-satisfying STC-labeling for $G^{\prime}$ with at most $k^{\prime}$ weak edges.

$(\Leftarrow)$ Conversely, let $\hat{L}=\left(S_{\hat{L}}^{1}, \ldots, S_{\hat{L}}^{c}, W_{\hat{L}}\right)$ be a $\Psi^{\prime}$-satisfying STC-labeling for $G^{\prime}$ such that $\left|W_{\hat{L}}\right| \leq k-\left|D_{E}^{\emptyset}\right|$. We define a $\Psi$-satisfying STC-labeling $L$ for $G$, with $\left|W_{L}\right| \leq k$. We first show that if a vertex in $\mathcal{N}\left(K^{\prime}\right)$ is connected to $K^{\prime}$ by many edges with the same strong color list, then these edges may receive the same strong color. To show the claim, consider a fixed vertex $v \in \mathcal{N}\left(K^{\prime}\right)$ and a set $K_{v} \subseteq K^{\prime}$ such that all edges in $E\left(\{v\}, K_{v}\right)$ have the same strong color list $\psi \neq \emptyset$ under $\Psi^{\prime}$.

Claim 12. If $\left|K_{v}\right| \geq\left|E\left(\{v\}, \mathcal{N}^{2}\left(K^{\prime}\right)\right)\right|$, then we can assume that $E\left(\{v\}, K_{v}\right) \subseteq S_{\hat{L}}^{i}$ for some $1 \leq i \leq c$. 
Proof. Since $K^{\prime}$ is a closed critical clique, we can assume by Lemma 3, that either all edges in $E\left(\{v\}, K_{v}\right)$ are weak or have the same strong color under $\hat{L}$. In the latter case, the claim is directly fulfilled. Thus, assume the first case holds, that is, $E\left(\{v\}, K_{v}\right) \subseteq W_{\hat{L}}$. Note that, whenever an edge $e \in E\left(\{v\}, K_{v}\right)$ forms an induced $P_{3}$ with another edge $e^{\prime}$, it follows that $e^{\prime} \in E\left(\{v\}, \mathcal{N}^{2}\left(K^{\prime}\right)\right)$. Let $i \in \psi$. We define a new labeling $P=\left(S_{P}^{1}, \ldots, S_{P}^{c}, W_{P}\right)$ for $G^{\prime}$ by

$$
\begin{aligned}
S_{P}^{i} & :=S_{\hat{L}}^{i} \cup E\left(\{v\}, K_{v}\right) \backslash E\left(\{v\}, \mathcal{N}^{2}\left(K^{\prime}\right)\right), \\
W_{P} & :=W_{\hat{L}} \backslash E\left(\{v\}, K_{v}\right) \cup\left(S_{\hat{L}}^{i} \cap E\left(\{v\}, \mathcal{N}^{2}\left(K^{\prime}\right)\right)\right), \text { and } \\
S_{P}^{j} & :=S_{\hat{L}}^{j} \text { for all } j \neq i .
\end{aligned}
$$

From $\left|K_{v}\right| \geq\left|E\left(\{v\}, \mathcal{N}^{2}\left(K^{\prime}\right)\right)\right|$ we conclude

$$
\begin{aligned}
\left|W_{P}\right| & =\left|W_{\hat{L}}\right|-\left|E\left(\{v\}, K_{v}\right)\right|+\left|S_{\hat{L}}^{i} \cap E\left(\{v\}, \mathcal{N}^{2}\left(K^{\prime}\right)\right)\right| \\
& \leq\left|W_{\hat{L}}\right|-\left|K_{v}\right|+\left|E\left(\{v\}, \mathcal{N}^{2}\left(K^{\prime}\right)\right)\right| \\
& \leq\left|W_{\hat{L}}\right| .
\end{aligned}
$$

Moreover, $P$ clearly is $\Psi^{\prime}$-satisfying. It remains to show that $P$ is an STC-labeling, which means that there is no induced $P_{3}$ containing an edge $e \in E\left(\{v\}, K_{v}\right) \subseteq S_{P}^{i}$ and another edge $e^{\prime} \in S_{P}^{i}$. As mentioned above, the edges in $E\left(\{v\}, K_{v}\right)$ only form an induced $P_{3}$ with edges from $E\left(\{v\}, \mathcal{N}^{2}\left(K^{\prime}\right)\right)$. By the construction of $P$, no edge from $E\left(\{v\}, \mathcal{N}^{2}\left(K^{\prime}\right)\right)$ belongs to $S_{P}^{i}$. Hence, $P$ is an STC-labeling.

Let thus $\hat{L}$ be such that the assumption of Claim 12 holds simultaneously for all eligible $v \in \mathcal{N}\left(K^{\prime}\right)$ and $K_{v}$. We define the labeling $L$ for $G$ by extending $\hat{L}$. We set $W_{L}:=W_{\hat{L}} \cup D_{E}^{\emptyset}$. Since $\left|W_{\hat{L}}\right| \leq k-\left|D_{E}^{\emptyset}\right|$, it holds that $\left|W_{L}\right| \leq k$. It remains to label all edges in $D_{E} \backslash D_{E}^{\emptyset}$. Let $u$ be some fixed vertex in $D_{V}$ and $v \in N(u)$ such that $\{u, v\} \notin D_{E}^{\emptyset}$.

Case 1: If $v \in K$, then the edge $\{u, v\}$ is an edge between two vertices of a critical clique. Since $\{u, v\} \notin D_{E}^{\emptyset}$, there is some $i \in \Psi(\{u, v\})$. We add $\{u, v\}$ to $S_{L}^{i}$. Clearly, $\{u, v\}$ satisfies the $\Psi$-list property. Since $\{u, v\}$ is not part of any induced $P_{3}$ this does not violate STC.

Case 2: If $v \in \mathcal{N}(K)$, then there is a set $Y \subseteq K^{\prime}$ containing at least $\left|E\left(\{v\}, \mathcal{N}^{2}(K)\right)\right|$ vertices distinct from $u$ such that $\Psi(\{v, y\})=\Psi(\{v, u\})$ for every $y \in Y$. Otherwise, $u$ would have been marked as important by Algorithm 1 , which contradicts the fact that $u \in D_{V}$. From Claim 12, we know that all edges in $E_{G^{\prime}}(\{v\}, Y)$ are elements of the same strong color class $S_{\hat{L}}^{i}$ for some $i \in\{1, \ldots, c\}$. We set $S_{L}^{i}:=S_{\hat{L}}^{i} \cup\{\{u, v\}\}$. Clearly, $\{u, v\}$ satisfies the $\Psi$-list property under $L$. Moreover, adding $\{u, v\}$ to $S_{L}^{i}$ does not violate STC: The only edges that form an induced $P_{3}$ with $\{u, v\}$ are the edges in $E\left(\{v\}, \mathcal{N}^{2}(K)\right)$. Now, since $E_{G^{\prime}}(\{v\}, Y) \subseteq S_{\hat{L}}^{i}$ and since $\hat{L}$ is an STC-labeling for $G^{\prime}$, none of these edges is contained in $S_{\hat{L}}^{i}$.

Consequently, $L$ is a $\Psi$-satisfying STC-labeling for $G$ with $\left|W_{L}\right| \leq k$.

We now consider instances that are reduced regarding Rule 1 . The following upper bound of the size of closed critical cliques is important for the kernel result.

Lemma 4. Let $(G, c, k, \Psi)$ be a reduced instance for EL-Multi-STC. For every closed critical clique $K$ in $G$ it holds that $|K| \leq \tau$. $\left|E\left(\mathcal{N}(K), \mathcal{N}^{2}(K)\right)\right|$.

Proof. We prove the lemma by having a closer look at the vertices that were not deleted by Algorithm 1 . Note that the algorithm is applied on every closed critical clique $K$ with $|K|>\tau \cdot\left|E\left(\mathcal{N}(K), \mathcal{N}^{2}(K)\right)\right|$. Every vertex that was not marked as important in Line 7 of the algorithm is deleted from $G$. Note that there are at most $\tau$ possible images $\psi$ of $\Psi: E \rightarrow 2^{\{1, \ldots, c\}}$. By Lines 7, 9, and 10 it holds that for every $v \in \mathcal{N}(v)$ the algorithm marks at most $\tau \cdot\left|E\left(\{v\}, \mathcal{N}^{2}(K)\right)\right|$ vertices of $K$. Consequently, there are at most

$$
\tau \cdot \sum_{v \in \mathcal{N}(K)}\left|E\left(\{v\}, \mathcal{N}^{2}(K)\right)\right|=\tau \cdot\left|E\left(\mathcal{N}(K), \mathcal{N}^{2}(K)\right)\right|
$$

marked vertices in $K$, since $\left\{E\left(\{v\}, \mathcal{N}^{2}(K)\right) \mid v \in \mathcal{N}(v)\right\}$ forms a partition of $E\left(\mathcal{N}(K), \mathcal{N}^{2}(K)\right)$. Hence, $|K| \leq \tau$. $\left|E\left(\mathcal{N}(K), \mathcal{N}^{2}(K)\right)\right|$ for every closed critical clique $K$ in $G$.

Theorem 5. EL-MuLTI-STC admits a problem kernel with at most $(\tau+1) \cdot 2 k_{1}$ vertices.

Proof. Let $(G=(V, E), c, k, \Psi)$ be a reduced instance for EL-MulTi-STC. We show that $|V| \leq(\tau+1) \cdot 2 k_{1}$.

The overall number of vertices in open critical cliques is at most $2 k_{1}$ [14]. Let $K$ be some closed critical clique. We now transform the graph $G$ into a modified graph $G^{\prime}$ in the following way. We replace every closed critical clique $K$ with a critical 
clique $K^{\prime}$ such that $\left|K^{\prime}\right|=\frac{|K|}{\tau}$. From Lemma 4 we know that $|K| \leq \tau \cdot\left|E\left(\mathcal{N}(K), \mathcal{N}^{2}(K)\right)\right|$ for every closed critical clique $K$ in $G$. Consequently, $\left|K^{\prime}\right| \leq\left|E\left(\mathcal{N}(K), \mathcal{N}^{2}(K)\right)\right|$ for every closed critical clique $K^{\prime}$ in $G^{\prime}$. As shown previously, this implies that the overall number of vertices in closed critical cliques in $G^{\prime}$ is at most $2 k_{1}$ [14, Proof of Theorem 1]. Hence, the overall number of vertices in closed critical cliques in $G$ is at most $\tau \cdot 2 k_{1}$, which gives us $|V| \leq 2 k_{1}+\tau \cdot 2 k_{1}=(\tau+1) \cdot 2 k_{1}$.

Recall that for any EL-Multi-STC instance $(G, c, k, \Psi)$ we have $\tau \leq 2^{c}-1$. Also, Multi-STC is the special case of ELMuLti-STC where every edge has the list $\{1,2, \ldots, c\}$, and thus $\tau=1$. These two facts imply the following.

Corollary 5. EL-MULTI-STC admits a problem kernel with at most $2^{c+1} k_{1}$ vertices. MuLTI-STC admits a problem kernel with at most $4 k_{1}$ vertices.

\section{Conclusion}

We have provided a first study of the computational complexity of MuLTI-STC and two of its generalizations. There are many interesting research questions that can be pursued in future work. Most importantly, it is open whether MuLTI-STC admits an algorithm with running time $2^{\mathcal{O}(n)}$, even when $k=0$. Let us remark that an algorithm with such a running time is also open for Edge Coloring: so far, it is only known that List-Edge Coloring, where a color list is given for each edge, admits no $2^{o\left(n^{2}\right)}$-time algorithm under the ETH. A first step to prove a lower bound for EDGE CoLoRING could be to prove that a vertex-list version of EDGE CoLoRING admits no $2^{o\left(n^{2}\right)}$-time algorithm as well.

For MulTI-STC with $c=2$, there exists a polynomial kernel for the parameter $k$ : There is a parameter preserving reduction to OdD Cycle Transversal [35] which has a polynomial kernel [28]. Then, since Multi-STC with $c=2$ is NP-hard and OdD CyCle TRANSVERSAL is in NP, there exists a polynomial kernel for Multi-STC. However, an interesting open question is if one could think of any direct problem kernelization for the parameter $k$.

A further direction of research could be to identify other applications of the structural parameter $k_{1}$ which is the vertex cover number of the Gallai graph. Even more generally, it seems interesting to study which graph problems become tractable when the Gallai graph of the input graph has a certain structure. To this end, one could further study relations between the structure of a graph and the structure of its Gallai graph. For example, is it possible to describe certain natural graph classes compactly via forbidden induced subgraphs of their Gallai graphs?

\section{CRediT authorship contribution statement}

Laurent Bulteau: Conceptualization. Niels Grüttemeier: Conceptualization, Writing - original draft preparation. Christian Komusiewicz: Conceptualization, Writing - original draft preparation. Manuel Sorge: Conceptualization, Writing - original draft preparation.

\section{Declaration of competing interest}

The authors declare that they have no known competing financial interests or personal relationships that could have appeared to influence the work reported in this paper.

\section{References}

[1] Akanksha Agrawal, Fine-grained complexity of rainbow coloring and its variants, in: Proceedings of the 42nd International Symposium on Mathematical Foundations of Computer Science, MFCS '17, in: LIPIcs, vol. 83, Schloss Dagstuhl - Leibniz-Zentrum für Informatik, 2017, pp. 60:1-60:14.

[2] Hans L. Bodlaender, Bart M.P. Jansen, Stefan Kratsch, Kernelization lower bounds by cross-composition, SIAM J. Discrete Math. 28 (1) (2014) $277-305$.

[3] Jianer Chen, Iyad A. Kanj, Ge Xia, Improved upper bounds for vertex cover, Theor. Comput. Sci. 411 (40-42) (2010) 3736-3756.

[4] Jianer Chen, Jie Meng, A $2 k$ kernel for the cluster editing problem, J. Comput. Syst. Sci. 78 (1) (2012) 211-220.

[5] Marek Cygan, Fedor V. Fomin, Lukasz Kowalik, Daniel Lokshtanov, Dániel Marx, Marcin Pilipczuk, Michal Pilipczuk, Saket Saurabh, Parameterized Algorithms, Springer, 2015

[6] Christopher P. Diehl, Galileo Namata, Lise Getoor, Relationship identification for social network discovery, in: Proceedings of the Twenty-Second AAAl Conference on Artificial Intelligence, AAAI '07, AAAI Press, 2007, pp. 546-552.

[7] Michael Dom, Daniel Lokshtanov, Saket Saurabh, Kernelization lower bounds through colors and IDs, ACM Trans. Algorithms 11 (2) (2014) 13:1-13:20.

[8] Rodney G. Downey, Michael R. Fellows, Fundamentals of Parameterized Complexity, Texts in Computer Science, Springer, 2013.

[9] Jörg Flum, Martin Grohe, Parameterized Complexity Theory, Texts in Theoretical Computer Science. An EATCS Series, Springer, 2006.

[10] Gallai Tibor, Transitiv orientierbare Graphen, Acta Math. Hung. 18 (1-2) (1967) 25-66.

[11] Petr A. Golovach, Pinar Heggernes, Athanasios L. Konstantinidis, Paloma T. Lima, Charis Papadopoulos, Parameterized aspects of strong subgraph closure, Algorithmica 82 (7) (2020) 2006-2038.

[12] Mark Granovetter, The strength of weak ties, Am. J. Sociol. 78 (1973) 1360-1380.

[13] Mark Granovetter, The strength of weak ties: a network theory revisited, Sociol. Theory (1983) 201-233.

[14] Niels Grüttemeier, Christian Komusiewicz, On the relation of strong triadic closure and cluster deletion, Algorithmica 82 (4) (2020) $853-880$.

[15] Niels Grüttemeier, Christian Komusiewicz, Nils Morawietz, Maximum edge-colorable subgraph and strong triadic closure parameterized by distance to low-degree graphs, in: Proceedings of the 17th Scandinavian Symposium and Workshops on Algorithm Theory, SWAT '20, in: LIPIcs, vol. 162, Schloss Dagstuhl - Leibniz-Zentrum für Informatik, 2020, pp. 26:1-26:17.

[16] Jiong Guo, A more effective linear kernelization for cluster editing, Theor. Comput. Sci. 410 (8-10) (2009) 718-726. 
[17] András Hajnal, Endre Szemerédi, Proof of a conjecture of P. Erdős, in: Combinatorial Theory and Its Applications, vol. 2, 1970, pp. 601-623.

[18] Ian Holyer, The NP-completeness of edge-coloring, SIAM J. Sci. Comput. 10 (4) (1981) 718-720.

[19] Wen-Lian Hsu, Tze-Heng Ma, Substitution decomposition on chordal graphs and applications, in: Proceedings of the 2nd International Symposium on Algorithms, ISA '91, in: LNCS, vol. 557, Springer, 1991, pp. 52-60.

[20] Hong Huang, Jie Tang, Sen Wu, Lu Liu, Xiaoming Fu, Mining triadic closure patterns in social networks, in: Companion Volume of the 23rd International World Wide Web Conference, WWW '14, ACM, 2014, pp. 499-504.

[21] Russell Impagliazzo, Ramamohan Paturi, Francis Zane, Which problems have strongly exponential complexity?, J. Comput. Syst. Sci. 63 (4) (2001) 512-530.

[22] Richard M. Karp, Reducibility among combinatorial problems, in: Complexity of Computer Computations, 1972, pp. 85-103.

[23] Henry A. Kierstead, Alexandr V. Kostochka, Marcelo Mydlarz, Endre Szemerédi, A fast algorithm for equitable coloring, Combinatorica 30 (2) (2010) 217-224.

[24] Athanasios L. Konstantinidis, Stavros D. Nikolopoulos, Charis Papadopoulos, Strong triadic closure in cographs and graphs of low maximum degree, Theor. Comput. Sci. 740 (2018) 76-84.

[25] Athanasios L. Konstantinidis, Charis Papadopoulos, Maximizing the strong triadic closure in split graphs and proper interval graphs, Discrete Appl. Math. 285 (2020) 79-95.

[26] Lukasz Kowalik, Juho Lauri, Arkadiusz Socala, On the fine-grained complexity of rainbow coloring, SIAM J. Discrete Math. 32 (2018) $1672-1705$.

[27] Lukasz Kowalik, Arkadiusz Socala, Tight lower bounds for list edge coloring, in: Proceedings of the 16th Scandinavian Symposium and Workshops on Algorithm Theory, SWAT '18, in: LIPIcs, vol. 101, Schloss Dagstuhl - Leibniz-Zentrum fuer Informatik, 2018, pp. 28:1-28:12.

[28] Stefan Kratsch, Magnus Wahlström, Compression via matroids: a randomized polynomial kernel for odd cycle transversal, ACM Trans. Algorithms 10 (4) (2014) 20:1-20:15.

[29] Van Bang Le, Gallai graphs and anti-Gallai graphs, Discrete Math. 159 (1-3) (1996) 179-189.

[30] Daniel Leven, Zvi Galil, NP completeness of finding the chromatic index of regular graphs, J. Algorithms 4 (1) (1983) 35-44.

[31] R. Niedermeier, Invitation to Fixed-Parameter Algorithms, Oxford Lecture Series in Mathematics and Its Applications, Oxford University Press, 2006.

[32] Fábio Protti, Maise Dantas da Silva, Jayme Luiz Szwarcfiter, Applying modular decomposition to parameterized cluster editing problems, Theory Comput. Syst. 44 (1) (2009) 91-104.

[33] Bruce A. Reed, Kaleigh Smith, Adrian Vetta, Finding odd cycle transversals, Oper. Res. Lett. 32 (4) (2004) 299-301.

[34] Polina Rozenshtein, Nikolaj Tatti, Aristides Gionis, Inferring the strength of social ties: a community-driven approach, in: Proceedings of the 23rd ACM SIGKDD International Conference on Knowledge Discovery and Data Mining, KDD '17, ACM, 2017, pp. 1017-1025.

[35] Stavros Sintos, Panayiotis Tsaparas, Using strong triadic closure to characterize ties in social networks, in: Proceedings of the 20th ACM SIGKDD International Conference on Knowledge Discovery and Data Mining, KDD '14, ACM, 2014, pp. 1466-1475.

[36] Liping Sun, Two classes of perfect graphs, J. Comb. Theory, Ser. B 53 (2) (1991) 273-292.

[37] Jie Tang, Tiancheng Lou, Jon M. Kleinberg, Inferring social ties across heterogenous networks, in: Proceedings of the Fifth International Conference on Web Search and Web Data Mining, WSDM '12, ACM, 2012, pp. 743-752.

[38] Craig A. Tovey, A simplified NP-complete satisfiability problem, Discrete Appl. Math. 8 (1) (1984) 85-89.

[39] René van Bevern, Oxana Yu. Tsidulko, Philipp Zschoche, Fixed-parameter algorithms for maximum-profit facility location under matroid constraints, in: Proceedings of the 11th International Conference on Algorithms and Complexity, CIAC '19, in: Lecture Notes in Computer Science, vol. 11485, Springer, 2019, pp. 62-74.

[40] Vadim G. Vizing, On an estimate of the chromatic class of a p-graph, Discret. Anal. 3 (1964) 25-30.

[41] Rongjing Xiang, Jennifer Neville, Monica Rogati, Modeling relationship strength in online social networks, in: Proceedings of the 19th International Conference on World Wide Web, WWW '10, ACM, 2010, pp. 981-990.

[42] Jaewon Yang, Jure Leskovec, Community-affiliation graph model for overlapping network community detection, in: Proceedings of the 12th IEEE International Conference on Data Mining, ICDM '12, IEEE Computer Society, 2012, pp. 1170-1175. 